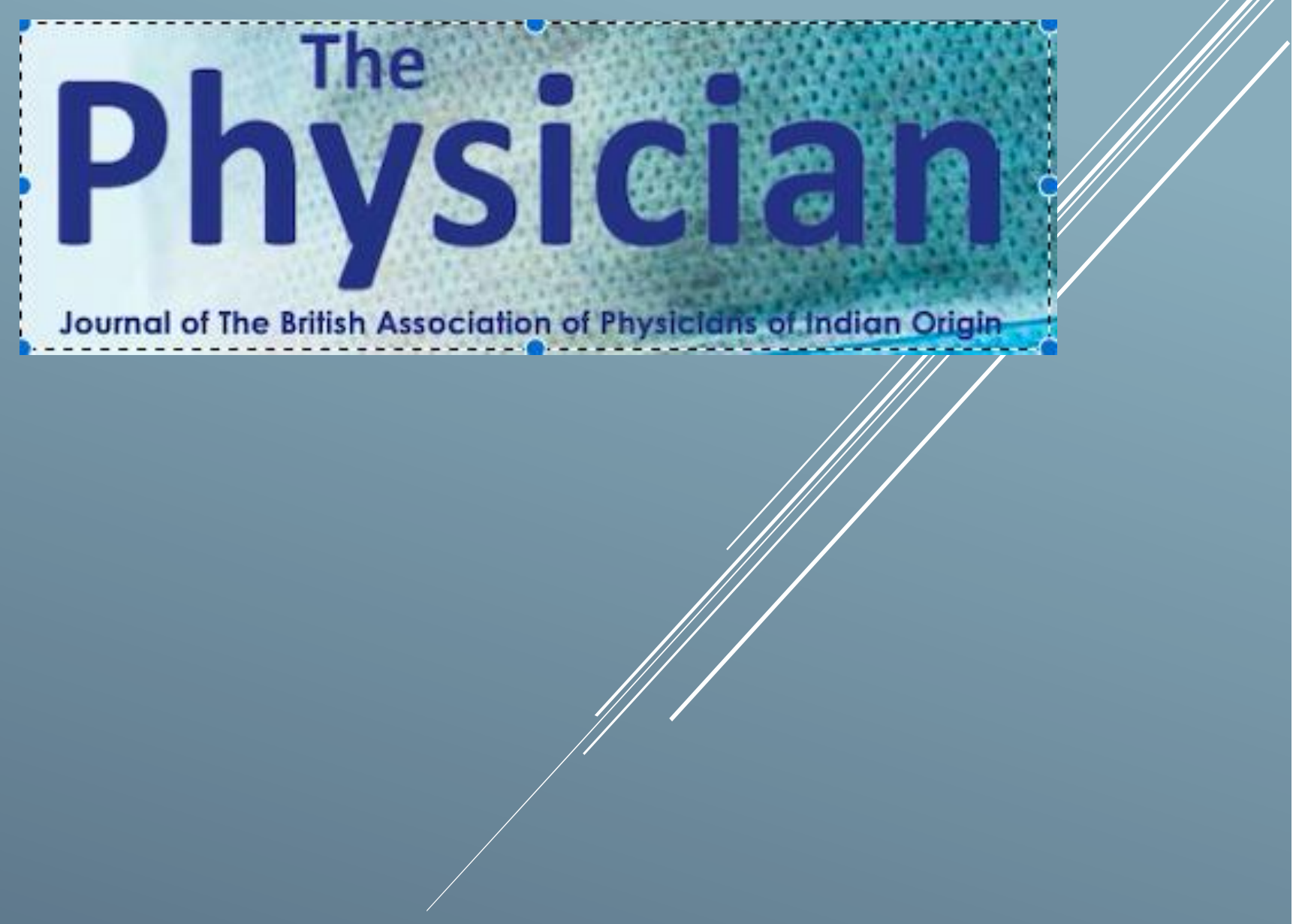

FORUM FOR

INTERDISCIPLINARY RESEARCH

IN MEDICAL SCIENCE \&

TECHNOLOGY

FIRMST 2020 | Moscow | Conference Abstracts 
Team

\title{
Forum for Interdisciplinary Research in Medical Science \& Technology
}

\author{
Moscow | Russian Federation
}

Conference 4-5 August 2020

FIRMST Secretariat

EAFO Educational \& Research Center Eurasian Oncology Program

Volokolomskoe Shosse, 1, Building 1, Office 606C

Moscow, Russian Federation,125 080

Somasundaram SUBRAMANIAN

FIRMST 2020 Convenor \& Founder

Russian Federation

https://www.firmst.tech

\section{The Vision}

FIRMST is a unique platform for interaction of world-class experts, internationally recognized physicians, surgeons and researchers, brilliant young scientists and students from all over the world in the field of Medicine \& Allied Sciences.

What makes FIRMST stand-out from the other Forums is its "the sky's the limit" approach. We strongly believe that great medical breakthroughs can be made only when a holistic approach is adopted. Here at FIRMST you may choose ANY topic of your choice from ANY discipline related to the medical field and initiate an open discussion about the same.

FIRMST aims to:

1. Sow the seeds of collaborative medical research into emerging minds in the various fields of healthcare.

2. Introduce students to a holistic approach towards medicine by encouraging open discussions between students and experts of various disciplines.

3. Encourage our youngsters to think outside just a single specialty and explore into other fields to find innovative and highly efficient solutions to the existing healthcare issues.

4. Bridge the gap between students, who do not get enough exposure or opportunities to be introduced to different specialties, subspecialties, and research at an early stage in their career, and the leaders who have the knowledge, expertise and resources ready at their disposal.

5. Emphasize that connections and interactions in the medical community are essential for collective progress. As such, we believe that FIRMST will enable the participants to forge those connections over time.

6. Introduce participants to advancements in healthcare, recent protocols for diagnosis and treatment, and to promote the modern means of thinking in the medical field - including interdisciplinary teamwork, the need for updating guidelines, and the need for continued improvement of individuals on a personal and professional level.

7. Create new Fellowship/Post-graduate or other educational opportunities for students, doctors, nurses and others in medicine and allied sciences from anywhere across the globe.

Conference Abstracts curated and peer reviewed by Bapio Institute for Health Research, Bedford, UK Professor JS Bamrah, Professor Soumit Dasgupta \& Dr Neeraj Bhala

https://www.bapio.co.uk/bihr 
Contents

TEAM

CONTENTS

NOVEL VIEW ON MEDICAL NURSING

WHAT'S HAPPENING WITH E-CIGARETTES IN IRELAND?

SMOKING IN SCHOOLS IN IRELAND

A TWO ARM PARALLEL DESIGN SUPERIORITY RANDOMIZED TRIAL COMPARING

PENICILLIN AND STANDARD OF CARE VS STANDARD OF CARE ALONE IN ACHIEVING

COMPLETE HEALING OF LEG ULCERS IN PATIENTS WITH CHRONIC VENOUS

HYPERTENSION.

CLINICAL AND RADIOLOGICAL FEATURES OF PNEUMONIA IN CHILDREN WITH

BRONCHIAL ASTHMA.

ANATOMY MENTORING PRACTICE AS A NEW PROMISING WAY FOR TEACHING

MORPHOLOGICAL SCIENCE

OPINIONS OF MEDICAL STUDENTS REGARDING ONLINE LEARNING: A

QUESTIONNAIRE BASED STUDY.

SAFETY OF HYDROXYCHLOROQUINE FOR COVID-19 PROPHYLAXIS IN HEALTHCARE

WORKERS - A SURVEY BASED STUDY.

KNOWLEDGE, ATTITUDE, AND PRACTICE TOWARDS SARS COV-2 AMONG MEDICAL

STUDENTS: A CROSS-SECTIONAL STUDY.

AN ASSESSMENT OF THE AWARENESS OF THE COVID-19 PANDEMIC: A

QUESTIONNAIRE BASED STUDY.

AN ADVERSE DRUG REACTION TO HYDROXYCHLOROQUINE : REVERSIBLE VISUAL

LOSS. A CASE REPORT.

"THE EFFECT OF LEFT NOSTRIL BREATHING (LNB) AND RIGHT NOSTRIL BREATHING

(RNB) ON CARDIOVASCULAR PARAMETERS": A SYSTEMATIC REVIEW.

A QUESTIONNAIRE BASED STUDY REGARDING THE KNOWLEDGE, ATTITUDE AND

PRACTICE OF SELF-MEDICATION AMONG UNDERGRADUATE MEDICAL STUDENTS.

COST EFFECTIVENESS OF ANTIHYPERTENSIVE DRUGS AVAILABLE IN INDIAN

MARKET.

ASSESSMENT OF KNOWLEDGE OF 2ND YEAR MEDICAL STUDENTS REGARDING PROMOTIONAL DRUG LITERATURE USING THE WORLD HEALTH ORGANISATION CRITERIA.

OPINIONS OF MEDICAL TEACHERS REGARDING ONLINE LEARNING: A

QUESTIONNAIRE STUDY.

COST EFFECTIVENESS ANALYSIS OF ORAL ANTIPLATELET DRUGS.

INTEGRATING MULTIDISCIPLINARY APPROACH WITH ADVANCED TECHNIQUES IN

DIAGNOSING DILEMMATIC BONE PATHOLOGIES.

MICROWAVE ENCEPHALOGRAPHY (MWENC) PROJECT - NEW TECHNOLOGY OF

THE BRAIN DIAGNOSTIC.

EFFICIENCY OF SANITATION WITH OZONIZED SOLUTION AFTER THE LIVER

ECHINOCOCCECTOMY

THE CYTOLOGICAL CHARACTERISTIC OF PERITONEAL FLUID AGAINST THE BACKGROUND OF INTRODUCTION OF OZONIZED PHYSIOLOGICAL SOLUTION IN EXPERIMENT

INFLUENCE OF OZONIZED SALINE SOLUTION ON THE COURSE OF PURULENT PERITONITIS IN THE EXPERIMENT: FOCUS ON THE CYTODYNAMICS OF PERITONEAL EXUDATE

PERIOPERATIVE ASSESSMENT OF DIAPHRAGMATIC MOVEMENT BY ULTRASOUND AND ITS INTERACTION WITH POSTOPERATIVE PULMONARY COMPLICATIONS FOLLOWING UPPER ABDOMINAL SURGERIES FOR CANCER: A LONGITUDINAL OBSERVATIONAL STUDY

IMMUNE EVASION OFFERS NOVEL INSIGHTS INTO THE EVOLUTIONARY TRAJECTORY OF UROLOGY'S MOST DEADLY CANCER.

WEARABLE ANGER MONITORING SYSTEM (WAMS).

LATANOPROST AND BIMATOPROST, SO SIMILAR, BUT WHICH IS BETTER? PREVENTION OF DAMAGE TO THE RECURRENT LARYNGEAL NERVES DURING THYROID SURGERY, CLINICAL MANIFESTATIONS AND TREATMENT PATIENTS. POSSIBILITIES OF SPERMATOGENESIS STIMULATION WITH LOW-LEVEL LASER THERAPY IN RED SPECTRUM IN MALE SECRETORY INFERTILITY. QUALITY OF LIFE AND BURNOUT IN PEDIATRIC DENTISTS: NO CAUSE FOR ALARM?
PARADIGMS OF LIVER TRANSPLANTATION FOR HEPATOCELLULAR CARCINOMA IN

1 THE REPUBLIC OF BELARUS.

1 THE OTHER SIDE OF PERSONAL PROTECTIVE EQUIPMENTS (PPES) : A

3 QUESTIONNAIRE BASE STUDY AMONG HEALTH CARE WORKERS WORKING IN

3 COVID SETUP.

4 DIETARY HABITS OF YOUNG PEOPLE IN STRESSFUL CONDITIONS DURING A PANDEMIC.

WEB SERVICE “QFAT" FOR CALCULATION INTAKE OF ESSENTIAL FATTY ACIDS: DEVELOPMENT AND APPLICATION.

5 KNOWLEDGE, ATTITUDE AND BEHAVIOR OF HEALTH CARE PROFESSIONALS TOWARDS DRUG PROMOTIONAL LITERATURE DISTRIBUTED BY PHARMACEUTICAL

5 COMPANIES IN A TERTIARY CARE TEACHING HOSPITAL. KNOWLEDGE, ATTITUDE AND PRACTICES OF RATIONAL USE OF MEDICINE

6 AMONGST POSTGRADUATES AND INTERNS IN A TERTIARY CARE TEACHING HOSPITAL.

QUALITY OF SLEEP PATTERN AMIDST THE COVID-19 PANDEMIC: A CROSSSECTIONAL OBSERVATIONAL STUDY

TELEPHONIC CLINICS: "A REVISIT DURING COVID-19"

A NOVEL TECHNIQUE OF MINIMALLY INVASIVE RETRORECTUS REPAIR OF VENTRAL

HERNIA- MODIFIED ETEP.

ANALYTICAL QUESTIONNAIRE BASED STUDY TO EVALUATE THE PATTERN OF

9 EATING DISORDER AMONGST MEDICAL FRATERNITY VS GENERAL POPULATION

DURING COVID 19 PANDEMIC AND ITS PILOT STUDY

27

THE CLINICAL SIGNIFICANCE OF LABORATORY BIOMARKERS OF AN ANTITUMOR IMMUNE RESPONSE.

ANALYTICAL QUESTIONNAIRE BASED STUDY TO EVALUATE THE PATTERN OF

EATING DISORDER AMONGST MEDICAL FRATERNITY VS GENERAL POPULATION

DURING COVID 19 PANDEMIC AND ITS PILOT STUDY. UNDERGRADUATE STUDENTS OF HEALTH SCIENCES UNIVERSITIES TOWARDS E-

11 LEARNING: NATIONAL QUESTIONNAIRE BASED OBSERVATIONAL STUDY 30 THE OCCULT RHEUMATIC SCOURGE: AN AUTOPSY ANALYSIS OF MISSED / SILENT RHEUMATIC HEART DISEASE.

12 CHALLENGES, ATTITUDE, AND PRACTICES TOWARDS E-LEARNING SESSIONS FOR MEDICAL EDUCATION BY FACULTIES DURING COVID 19 PANDEMIC: A NATIONAL 12 QUESTIONNAIRE BASED OBSERVATIONAL STUDY IN TERTIARY CARE TEACHING

13 HOSPITALS KNOWLEDGE, ATTITUDE AND PRACTICE OF SELF MEDICATION DURING COVID-19

14 PANDEMIC: A QUESTIONNAIRE BASED STUDY KNOWLEDGE AND AWARENESS ABOUT COVID-19 AMONG GENERAL POPULATION

VISUAL ACUITY CHANGES ASSOCIATED WITH SHUNT MALFUNCTION AND OPTIC

32

I C4 LEVELS AS A PREDICTOR OF FLARES AND ADVERSE PREGNANCY OUTCOMES IN

15 PREGNANT WOMEN WITH SYSTEMIC LUPUS ERYTHEMATOSIS 34 E-CIGARETTES ALL SMOKE AND NO FIRE? PLEIOTROPIC EFFECTS OF STATINS - A MINI LITERATURE REVIEW. 36 SUCCESSFUL EXPERIENCE OF TREATMENT OF THE PRIMARY MULTIPLE MALIGNANT TUMOURS (PMMT): RETINOBLASTOMA, OSTEOSARCOMA. CLINICAL CASE. 36 CLINICAL SIGNIFICANCE OF THE MOST COMMON MUTATIONS IN THYROID CANCER.

17 A COST VARIATION ANALYSIS OF VARIOUS BRANDS OF ORAL ANTI-DIABETIC DRUGS CURRENTLY AVAILABLE IN INDIAN PHARMACEUTICAL MARKET.

17 DEUS EX MACHINA: SYSTEMATIZATION OF CURRENT INFORMATION ON

18 HELICOBACTER PYLORI INFECTION.

19 TREATMENT OF ADVANCED LARYNGEAL CANCER.

THE ROLE OF THE KI-67 MARKER IN ASSESSING THE QUANTITATIVE DEGREE OF NCY OF BREAST CANCER IN PET / ROLE OF ANGIOTENSIN RECEPTORS BLOCKERS IN TREATMENT OF COVID-19. 40 RESISTANCE TO ANTIBIOTICS OF STAPHYLOCOCCUS SPECIES ISOLATED FROM WOMEN WITH RECURRENT MISCARRIAGE OF PREGNANCY. BRAIN STIMULATION - AN AVENUE FOR THERAPY OF MEMORY DISORDERS AND IMPROVISING MEMORY 


\section{FÎ́RMST}

4-5 Aug 2020 | Moscow

Physicianjnl.net

THE POSSIBILITY OF IMPROVING MEMORY BEYOND NORMS AND PROVISION OF

AN AVENUE OF THERAPY FOR MEMORY DISORDERS.

THE UNANSWERED BIG WHY

US DIAGNOSTICS OF CYSTIC LESIONS OF THE NECK.

SONOGRAPHY OF CYSTIC LESIONS OF THE NECK.

INVESTIGATING THE ASSOCIATION BETWEEN OXYTOCIN PATHWAY

POLYMORPHISMS AND THE LEVEL OF SOCIAL FUNCTIONING IN SCHIZOPHRENIA

PATIENTS.

45

THE ROLE OF 18F-FDG PET/CT FOR THE PREDICTION OF RADIOIODINE THERAPY

RESPONSE IN PATIENTS WITH METASTATIC DTC

THE 'THOUGHT MODEL' OF SPIRITUALITY TO SOLVE COMPLEXITIES, CONFLICTS,

AND AMBIGUITIES IN TRAINING AND EDUCATION OF SPIRITUAL DIMENSION OF

HEALTH (SPIRITUAL HEALTH) IN MODERN PUBLIC HEALTHCARE SYSTEMS OF

CULTURALLY AND RELIGIOUSLY DIVERSE SOCIETIES.

46

EVALUATION OF VITAMIN D STATUS IN WOMEN DEPENDING ON THE AGE, BMI

VALUES, DURATION OF DAYLIGHT HOURS AND IN POLYCYSTIC OVARIAN

SYNDROME PATIENTS

NORMAL MR SPECTROSCOPIC MAP OF THE HEALTHY BRAIN

DEVELOPMENT OF A NEW METHOD OF AORTIC VALVE DECELLULARIZATION USING

THE SUPERCRITICAL EXTRACTION TECHNOLOGY.

SIMULTANEOUSLY DEVELOPED UTERINE CARCINOSARCOMA AND ENDOMETRIOD

OVARIAN CARCINOMA IN 21-YEAR-OLD WOMAN. 


\section{FÎ́RMST}

4-5 Aug 2020 | Moscow

FIRMST20_AB_78

Non-Clinical Sciences - Healthcare and Management

\section{NOVEL VIEW ON MEDICAL NURSING}

\section{Andrey TARABAROV1}

1I.M. Sechenov First Moscow Medical University, Moscow, Russia.

Russia, like most developing and developed countries, is an initiator and participant in various scientific research in the medical field. An example of such work - clinical trials of new or already registered drugs, if the method of their use differs from the approved one. According to the ICH GCP p. 4.1.5, p. 4.2.3 physicianresearcher may involve only personnel of the required qualifications and in an amount sufficient to comply with all the conditions of the research protocol.

The purpose of this article is to highlight the necessity of scientific training for nurses for participation in clinical research and work that requires an increased level of competence and a greater depth of knowledge.

Following the interviews of two principal researchers a list of the main requirements for nursing personnel involved in research has been compiled.

These requirements include:

1. Controlling and informing the investigating physician about the presence (arrival) of a suitable patient in the hospital.

2. Maintenance of non-medical documentation (questionnaires / brochures / checklists)

3. Accurate and complete execution of appointments in accordance with the research protocol (there are situations when the number of participating personnel is not able to ensure the execution of all appointments on time, which casts doubt on the correctness of the research)

4. Careful documentation of any and all of events occurring with patients in the study

5. To observe discipline and coordination (disclosure of inaccurate information, misleading the patient, etc.)

6. Control of delivery of the investigational drug to the facility (if the investigator / organization require such) 7. Full awareness of the subject of research, the principles of its work and other requirements.

8. Assistance to the physician in maintaining documentation, if the level of competence permits.
Based on the parameters above, nurses involved in research are required to have a high level of professionalism and understanding of the importance of research. Also, narrow specialization in the field of clinical trials, which in the realities of an ordinary department of a public or commercial hospital is almost impossible.

Proposed solution to this issue includes the introduction of advanced training courses similar to ones that physicians pass. As such, it is recommended for nursing staff to be aware of the international rules for conducting clinical trials, regulatory documents governing the conduct of clinical trials, be aware of the standards of planning, organization, conduct, monitoring, audits, documenting clinical trials, analyzing and presenting their results.

FIRMST20_AB_77

Non-Clinical Sciences-Tobacco Control

\section{WHAT'S HAPPENING WITH E-CIGARETTES IN IRELAND?}

\section{Luke CLANCY1, Joan HANAFIN1, Sheila \\ KEOGAN1, Salome SUNDAY1}

1Technological University Dublin, Dublin, Ireland

Introduction: E-cigarette use has increased rapidly worldwide since 2013 and there have been worrying increases in the use of e-cigarettes by adolescents and young people. In Ireland, smoking prevalence has declined significantly in recent decades but the availability of e-cigarettes in Ireland in recent years has led to

concerns about the Tobacco Endgame. We examine changes in e-cigarette use among 15-16 year olds in Ireland between 2015 and 2019.

Materials and Methods: We use data from 2 waves (2015, 1,472 students and 2019, 1,949 students) of the Irish wing of the European Schools Project for Alcohol and Other Drugs (ESPAD), a cross-sectional survey that, every four years, collects comparable data on substance use among European students aged 15 and 16 years, including on e-cigarette use.

Results: More students report using e-cigarettes in 2019 than in 2015, and the use of e-cigarettes among students is now more common than cigarette 


\section{FÎ́RMST}

4-5 Aug 2020 | Moscow

smoking. In 2019, almost four in 10 students (39\%) had tried e-cigarettes and almost one in $6(16 \%)$ were current users, up from $23 \%$ and $10 \%$ respectively in 2015. In 2019, boys (46\%) were more likely than girls $(33 \%)$ to have tried e-cigarettes and also to be current users ( $22 \%$ vs $12 \%)$. When asked about their reasons for trying e-cigarettes, about two-thirds (63\%, 66\%) said that it was "out of curiosity" in both 2015 and 2019 , and $21 \%$ and $29 \%$ respectively said that it was because their friends offered it. In 2015, 17\% said that it was to quit smoking while in 2019 , only $3 \%$ gave that reason. Asked about their tobacco use when they first used an e-cigarette, in 2015 33\% had never smoked tobacco but this had doubled to 68\% in 2019.

Conclusion : E-cigarette use among 15-16 year olds in Ireland has increased more than 50\% between 2015 and 2019, with boys more at risk than girls. Of particular concern is the significant increase in the numbers of young people using e-cigarettes who have never smoked tobacco. Policy and legislative implications include provision of health education for young people about e-cigarettes, consideration of ecigarette cessation programmes for adolescents, and the extension of tobacco control legislation regarding minors to be extended to include e-cigarettes.

Keywords: E-cigarettes, tobacco control, adolescents, health education, trend

FIRMST20_AB_76

Non-Clinical Sciences-Tobacco Control

\section{SMOKING IN SCHOOLS IN IRELAND}

Luke CLANCY1, Joan HANAFIN1, Sheila KEOGAN1, Salome SUNDAY1

1Technological University Dublin, Dublin, Ireland

Introduction: Smoking remains a notable issue for young people. The health effects of long-term use are well known and very serious, causing 8 million deaths worldwide annually from Cancer, Heart attacks, COPD and many other diseases In Ireland there is a national determination to become tobacco free by 2025 by which is meant a prevalence of smoking of less than $5 \%$. For this to be possible we need no new smokers to be added and most smokers to stop. Smoking in 16- year-olds has been reducing over the last 25 years and we need to monitor smoking in young people to see if we can eradicate smoking in youth.

Methods: In May 2019 using pen and paper and the standardised ESPAD survey instrument we surveyed a stratified random sample of 1949 students born in 2003. $49 \%$ were male and $51 \%$ were female.

Results: $32 \%$ of respondents had tried smoking and $14 \%$ were current smokers, with $5 \%$ smoking daily. The majority (63\%) of students reported starting to smoke at age 14 or 15 . Access to cigarettes was reported as easy by $61 \%$ of students. Smoking was statistically significantly $(p=0.001)$ associated with truancy and lower grades, perceived relative wealth, lower parental education, parental monitoring, rule setting, support, relationship with parents, peer smoking, alcohol, cannabis and other substance use. A trend analysis showed that, despite a reduction of over twothirds since 1995 (the second largest decline of any of the seven major indicators of the ESPAD survey in Ireland), slightly more students reported smoking in 2019 than in 2015, and this was pronounced for boys. In Ireland, smoking among these 15-16-year olds was greatly reduced to $14 \%$ in 2019 . This represents a reduction of over two-thirds (66\%) since 1995.

Conclusions: While these results are encouraging the failure of prevalence to fall since 2015 is disappointing. It suggests that greater efforts are needed if our young people are to be tobacco free by 2025 . More interventions focused on youth as well as all the known successful interventions that are effective in adults are needed.

Keywords: Smoking, schools, prevalence, trend, tobacco

FIRMST20_AB_75

Clinical Sciences - Surgery 


\section{FÎ́RMST}

4-5 Aug 2020 | Moscow

A TWO ARM PARALLEL DESIGN SUPERIORITY

RANDOMIZED TRIAL COMPARING PENICILLIN AND STANDARD OF CARE VS STANDARD OF CARE ALONE IN ACHIEVING COMPLETE HEALING OF LEG ULCERS IN PATIENTS WITH CHRONIC VENOUS HYPERTENSION.

Neeraj KUMAR1, Anita DHAR1

1All India Institute of Medical Sciences, New Delhi, India

Background: Chronic ulcers are one of the most difficult diseases to treat with socioeconomic impact, due to pain, recurrence $\&$ morbidity. Compression therapy with surgical \& endovascular procedure is required for management. Venous hypertension leads to oedema followed by microlymphangiopathy \& dermato-lymphangio-adenitis (DLA), attributed to recurrence of symptoms \& eventually ulcers. Role of Injection (Inj.) benzathine penicillin has been well established in treating lymphangitis. However, its role in venous ulcers hasn't been studied before.

Objective: Aim was to compare reduction in ulcer size, recurrence rate, pain score $\&$ improvement in quality of life(QOL) in patients receiving standard care(SoC) for chronic venous ulcer with \& without concomitant treatment with Inj. Benzathine penicillin 1.2 mega units intramuscular (IM) every 3 weekly for 6 months.

Design and Methods: Present randomized, two-arm superiority trial was conducted among patients aged 18-70 years with pregnancy, Acute DVT, Peripheral Arterial Disease, T2DM, penicillin allergy, etc. excluded. Participants were randomly recruited into two arms, control arm received SoC \& study arm received Inj Benzathine Penicillin with SoC. SoC included moist occlusive dressing, flavonoids, Short stretch bandages/ Stockings. SoC also included interventional treatment included Endovenous Laser Ablation/ Sclerotherapy/Subfascial Endoscopic Perforator Surgery/ Stripping \& ligation, etc. Patients were followed up weekly for 1 month, followed by monthly for 6 months.

Results: 66 patients with total 103 venous ulcers, baseline characteristics of both groups were statistically similar, however, ulcers did not follow a normal distribution at presentation \& there was a significant difference between mean ulcer size among the groups $(p=0.04)$. Compared with control $\operatorname{arm}(\mathrm{N}=32$, 49 ulcers $)$, the study $\operatorname{arm}(\mathrm{N}=34,54$ ulcers $)$ was associated with a higher number of healed ulcers. Mean size of ulcers decreased in both the arms with a statistically significant difference in study $\operatorname{arm}(p=0.03)$. Patients of study arm had lesser no. of recurrence. Pain control was improved in both arms, with significant difference favouring study group $(p=0.001)$. Significant difference in various domains of QoL was noted $(p=0.001)$. There were decreased attacks of DLA and limb swellings in both groups. With much more improved results in the study arm $(p=0.001)$.

Conclusions: Inj. Benzathine Penicillin administration along with SoC among chronic venous recurrent ulcers had beneficial effects on ulcer healing, QoL, recurrence, DLA \& pain.

Keyword: Venous ulcer, penicillin, venous diseases, randomized trial

FIRMST20_AB_74

Clinical Sciences - Nutrition

\section{CLINICAL AND RADIOLOGICAL FEATURES OF}

PNEUMONIA IN CHILDREN WITH BRONCHIAL

ASTHMA.

\section{Onkar HALMARE1, Tatyana VIVTANENKO1}

1FSBEI HE ORGMU of the Ministry of Health of Russia, Orenburg, Russia

Aim: To determine clinical and radiological features of pneumonia in children with bronchial asthma.

Materials and Methods: Under observation, children with pneumonia ( $n=59$ ) aged from 9 months to 17 years old, who received inpatient treatment in the pulmonology department of the children's allergocenter Regional clinical hospital No. 2 of Orenburg in 2015. All children were divided into 2 groups. The first group included 32 children (20 boys, 12 girls) with pneumonia and bronchial asthma (group A), the second group include - 27 children ( 20 boys and 7 girls) with pneumonia without bronchial asthma (group P).

Results: In $44.4 \%$ of cases among children with pneumonia (12 children), bronchial obstruction was regarded as a complication of pneumonia, while bronchial obstruction was repeated in $37 \%$ of cases (10 patients). 6 patients $(18.7 \%)$ had mild intermittent asthma and 4 (12.5\%) mild persistent asthma, 6 (18.75\%) moderate asthma, and 4 (12.5\%) had severe asthma.Prior to the anamnesis data, it was revealed 


\section{FÎ́RMST}

4-5 Aug 2020 | Moscow

that more often pneumonia was detected in the first children in the family - 62.5\% in group " $A$ " $(n=20), 51$. $8 \%$ in group " $P$ " $(n=14)$. The first time pneumonia was diagnosed in a group of children without bronchial asthma in $74 \%$ of cases (n-15); in $46.8 \%$ in group " $A$ " (n $=20$ ) in $74.07 \%$ in group $P$, respectively, and among children with asthma pneumonia was repeated $53,1 \%$ ( $n=17$; in group " $P$ " - $25.9 \%, n=7$ ). Severe pneumonia reported in group " $A$ " \% $(n=0)$ and in group " $P$ " $7.4 \%$ $(n=2)$ For children with pneumonia without bronchial asthma, cytomegalovirus, herpetic, candidal infections are more typical, for children with asthma, parasitic infection. food, drug allergies, seasonal allergic rhinitis is more often detected in children with bronchial asthma.

Conclusions: Localization, the extent of infiltration of lung tissue does not depend on the presence of bronchial asthma, more often pneumonia is rightsided and segmental, which is determined by the anatomical features of the structure of the bronchi. The development of pneumonia is influenced by the initial state of the child - a burdened allergic history, iron deficiency, chronic persistent infection and parasitic invasions, bronchial asthma contributes to the development of pneumonia, which is confirmed by monocytosis and the absence of inflammatory reaction from the peripheral blood, and increase in the recurrence of pneumonia in the group of children with bronchial asthma.

Keywords: Bronchial asthma, pneumonia, children, immunoglobulin $\mathrm{E}$, chest $\mathrm{x}$-ray

Conflict of Interests: none to declare

FIRMST20_AB 73

Non-Clinical Sciences - Anatomy

\section{ANATOMY MENTORING PRACTICE AS A NEW} PROMISING WAY FOR TEACHING MORPHOLOGICAL SCIENCE

Yasmin EL-TARAVI1, Erika, PORUBAEVA1, 1Sechenov University, Moscow, Russian Federation.

Introduction: Russian students learn human anatomy during 1st academic year as a separated course. Traditionally, the anatomy of the human body as the university course is associated with the number of complexities in the 1st year students. Pupils' decreased attentiveness in the period of adaptation for student's life causes the necessity for novel teaching strategies in anatomy. Thus, our study considers the benefits and drawbacks of new mentoring practice - mentorship - used in Sechenov University.

Materials \& Methods: Our new mentoring technique have been prepared and developed by the group of professors together with the most diligent students. The mentorship has been provided at the anatomy department of the Sechenov University in 2018-2019 academic year. We collected the results of final anatomy examination from 5 mentored and 5 nonmentored groups to evaluate the results of using this technique.

Results: Modern mentoring practice in anatomy department implies mentors teaching their coeval through education plan. The mentors are the most talented and motivated 1st year student with excellent marks during the first term-time. There are no special requests for students who will being trained; therefore, everyone could be taught within this type of program. Twenty chosen educators elaborate lesson plan in a special block of themes, and two testing controls both for student's knowledge assessment and collecting statistics. Our intermediate results received during 2018-2019 academic years show the increased marks in five taught groups from medical, pediatrics, and foreign students' faculties. Furthermore, all the mentors from scientific faculty demonstrate great individual advancement.

Conclusions: Now we're going to collect and analyze examination results for. We've created the questionnaire which includes 45 question separated to 6 block to evaluate students feedback for the program. However, we're convinced that our novel mentoring experience becomes necessary for all the student to recognize the responsibility for not's only their own education, but both for qualify teaching their coeval. Finally, this unusual experience will foster awareness of student leadership attributes.

Keywords: Mentorship, mentoring practice, medical teaching, anatomy teaching, novel teaching strategies, academic performance.

Conflict of interests: There is no conflict of interests 


\section{FÎ́RMST}

4-5 Aug 2020 | Moscow

FIRMST20_AB_72

Non-Clinical Sciences - Pharmacology

OPINIONS OF MEDICAL STUDENTS REGARDING

ONLINE LEARNING: A QUESTIONNAIRE BASED STUDY.

Pramila YADAV1, Dwija DESAI1, Pradnya DEOLEKAR1, 1Dr. D.Y. Patil University, School Of Medicine, Navi Mumbai, India

Background: The impact of COVID-19 pandemic is profound not only on physical health but also on routine teaching worldwide as a result of containment measures being taken for its prevention. Medical education is also affected as a result of changes in current teaching. As there is uncertainty about duration of this pandemic and social distancing measures are needed for long, hence, education of future doctors requires intense and prompt attention. Online teaching is a cheap and feasible method which helps to gain knowledge.

With our study we would like to highlight the issues related to changes in current teaching

methodology in the medical students in a medical College in Navi Mumbai.

Materials And Method: A simple survey based cross sectional study design using electronic questionnaire on Google Forms ${ }^{\mathrm{TM}}$, then evaluated and validated by independent expert. RESULTS: After 3 months of online teaching program, perceptions of first year to fourth students ( 250) were asked online using a questionnaire constituting of $33.6 \%$ from 1 st prof, $17.2 \%$ from 2nd prof,33.6\%,from 3rd prof,15.6\% from Final prof Students. $69.2 \%$ thought online learning was somewhat beneficial. Majority felt ward teaching, practical and clinical teaching is grossly deficient. $63.2 \%$ were of the opinion that online learning should be for minimum of 2 hours..52\% had the internet network good enough in their locality to serve the purpose of long hours of online class. Almost half to them felt use of online learning should be limited till the pandemic situation normalizes.

Conclusion: in contrast to most other literature which felt that online teaching, cheap and feasible method which helps to gain knowledge, maintaining routine and improving morale of teachers and student.
Keywords: Tools of online learning, Internet difficulties, Lack of concentration, Difficulties of Clinical learning.

Conflict of interests: None

\author{
FIRMST20_AB_71 \\ Non-Clinical Sciences - Pharmacology
}

\section{SAFETY OF HYDROXYCHLOROQUINE FOR COVID-19 PROPHYLAXIS IN HEALTHCARE WORKERS - A SURVEY BASED STUDY.}

\section{Pramila YADAV1, Gauravi RANADE1, Pradnya DEOLEKAR1}

1D Y Patil University, School of Medicine, Navi Mumbai, India.

Aim: To study the safety profile of hydroxychloroquine used for COVID-19 prophylaxis in healthcare workers

Introduction: The sudden outbreak and uncontrollable spread of the SARS-CoV-2 causing the deadly disease COVID-19 was officially declared a pandemic by the WHO in March 2020. Healthcare workers in contact with COVID-19 positive patients are at an elevated risk of contracting the infection.

With rapid rise in number of cases in India, the National Task Force for COVID-19 has recommended the use of HCQ for COVID-19 prophylaxis in healthcare workers. Although, its use as a prophylactic drug in asymptomatic individuals and its related adverse event profile is still unknown. Research on chemoprophylaxis is still lacking. This study aims to assess the adverse effects experienced by healthcare workers taking Hydroxychloroquine as prophylaxis.

Materials And Method: The conducted study was a cross sectional, observational based study amongst healthcare workers (HCW) in India exposed to COVID19 positive patients. A self-designed validated electronic questionnaire was circulated and responses about the demographics, pre-existing co-morbidities, any possible adverse effects of use of HCQ used were recorded and analysed. 
Results: From the 125 responses, majority i.e. $86.4 \%$ being doctors, $51.2 \%$ were from ages $41-60$ years with $64 \%$ being males. $84.8 \%$ had direct contact with COVID-19 positive patients. $38.4 \%$ responders have been taking HCQ for 5-7 weeks and 33.6\% have been taking it for 8-11 weeks. $35.2 \%$ responders experienced at least one adverse effect with most common adverse effects being $38 \%$ gastrointestinal followed by $22.4 \%$ experiencing headache and lethargy, and $11 \%$ experiencing chest pain. Before prescribing, 45\% responders were prescribed an ECG test, questioned for any pre-existing cardiac arrhythmias, allergies, G-6-PD deficiency and if they were taking any other medication. Co-morbidities observed included hypertension (15.2\%), respiratory disorders (2.4\%) and diabetes mellitus (2.4\%). In spite of taking HCQ for COVID-19 prophylaxis, $9.6 \%$ contracted the disease.

Conclusion: A higher incidence of adverse events was observed, especially gastrointestinal. Monitoring prior and during prophylaxis was inadequate even amongst those with cardiovascular disease and risk-factors. However, no serious cardiovascular events were reported.

Keywords: Hydroxychloroquine, healthcare workers, prophylaxis, COVID-19

Conflict of interests: None

FIRMST20_AB_70

Research Methodology \& Implementation - Basic Research

\section{KNOWLEDGE, ATTITUDE, AND PRACTICE TOWARDS} SARS COV-2 AMONG MEDICAL STUDENTS: A CROSSSECTIONAL STUDY.

Pramila YADAV1, Oorja Gyan Prakash SHARAF1, Pradnya, DEOLEKAR1,

1DY Patil University School of Medicine, Navi Mumbai, India.

Introduction: In December 2019, SARS- CoV-2 was recognized which caused serious illness and numerous deaths. The ultimate scope and effect are unclear at present with the situation rapidly evolving. Medical Students are directly or indirectly related to such epidemics. Being the pillars upholding the future of healthcare, they are expected to have a higher level of knowledge and better attitude towards such diseases. Hence our objective was to assess the level of knowledge, attitude, and practice of medical students towards this disease.

Methods: Online descriptive cross-sectional study was conducted where a self-designed questionnaire in the form of Google forms was developed and sent to students studying in the medical colleges from the different cities. The Google form link was sent through social media platforms like WhatsApp.

Results: Out of the total respondents ( $n=163), 30.7 \%$ were male and $69.3 \%$ were female, with $60.1 \%$ as hostellers. Highest responses were from 1st year $(30.7 \%)$ \& the least were from 2nd year students (19\%). Almost all were aware about COVID-19 and its mode of spread. While $81.3 \%$ could correctly define "close contact", $87.7 \%$ strongly agreed that an asymptomatic COVID-19 patient can spread the disease. $74.2 \%$ were aware of the investigations including tests, samples and medicines used for COVID-19. 60.1\% strongly agreed that masks are essential for healthy people. $27.3 \%$ strongly agree \& $27.3 \%$ were neutral with lockdown in controlling spread of diseases. $59.5 \%$ were hopeful of the vaccine's approval $73.6 \%$ considered taking it. While $27 \%$ feared the medical profession due to pandemic, $61.3 \%$ believed that the school students will consider taking the medical profession in the future.

Conclusion: The majority of the students had good knowledge, positive attitude, and sufficient practice. Such kind of Studies can help to make public-health policies targeting the specific groups with low KAP and increase their KAP through well-planned, appropriate and tailored strategies.

Keywords: knowledge, attitude, and practice, COVID19, Medical students.

Conflict of interests: None

FIRMST20_AB_69 


\section{FÎ́RMST}

4-5 Aug 2020 | Moscow

Non-Clinical Sciences - Pharmacology

AN ASSESSMENT OF THE AWARENESS OF THE COVID19 PANDEMIC: A QUESTIONNAIRE BASED STUDY.

Pramila YADAV1, Kanish CHANDRASHEKHAR1, Pradnya, DEOLEKAR1

1Dr.D Y Patil University School of Medicine,Navi Mumbai, India

Introduction: COVID-19 which was declared as a pandemic by the WHO in March 2020 has caused a massive public reaction. Pandemics can lead to heightened levels of stress; which in turn cause anxiety. The country went into lockdown on March 25, making it the largest lockdown in the world, restricting 1.3 billion people. Despite general awareness programs conducted by the state and central governments, there are still many false beliefs among the general public of India due to circulation of various misconceptions online about the spread of novel coronavirus, and prevention \& treatment of COVID-19. The present questionnaire study is intended to assess the awareness of the COVID-19 pandemic amongst general population.

Aim: To assess the awareness of the general public during the COVID-19 pandemic

Method: A web-based cross-sectional survey was conducted amongst 745 individuals.A 15-item questionnaire was developed, validated, and used for the study, after previously being approved by the faculty ethics committee.The questionnaire was randomly distributed among the public using Google forms through social media networks.

Result: Feedback responses were received from 758 participants from different family backgrounds, education level, occupation etc., as well as hailing from different parts of the country. Maximum around $82 \%$ of them received their updates from the news channels. The media updates were followed regularly by $48 \%$ individuals with $43 \%$ feeling that newspapers affected the way people think. $47 \%$ felt that there was a fear pandemic being spread through media, while $5 \%$ disagreed. $27 \%$ were afraid for their lives due to COVID, while $22 \%$ were scared of COVID because of being isolated from their families. Around $80 \%$ knew the type of people at high risk. $49 \%$ agreed that there was a stigma associated with the disease. $62 \%$ people started exercising daily at home, since the pandemic.
$52 \%$ agreed that news of the vaccine has helped reduce their fear of COVID-19.

Conclusion: Majority of participants are in update with recent happenings of covid. There is considerable fear about the pandemic. This survey would help the health care workers and administration to generate better awareness programs.

Keywords: COVID, awareness, questionnaire, assess, pandemic

Conflict of interests: None

FIRMST20_AB_68

Non-Clinical Sciences - Pharmacology

AN ADVERSE DRUG REACTION TO

HYDROXYCHLOROQUINE : REVERSIBLE VISUAL LOSS.

A CASE REPORT.

Pramila YADAV1, Pradnya DEOLEKAR1, Aakansha SINGH1

1Dy Patil School of Medicine, Navi Mumbai, India

Introduction: Hydroxychloroquine (HCQ) is a more soluble and less toxic metabolite of chloroquine with fewer concerns about drug interactions. Since it is affordable and widely available there had been a growing interest in the use of $\mathrm{HCQ}$ as potential treatments for Severe Acute Respiratory Syndrome Coronavirus-2 (SARS-CoV-2) till novel, specific treatments become available. Current data however does notrecommend for or against the use of these drugs. HCQ is currently prescribed to all COVID positive patients in India. A summary of the FDA review of safety issues ( July 20) with the use of Hydroxychloroquine includes reports of serious heart rhythm problems and other safety issues, including blood and lymph system disorders, kidney injuries, and liver problems and failure. We present a single case report of a 49-year-old female presenting as an asymptomatic COVID developing reversible visual loss with doses of HCQ taken for COVID for 5 days.

Discussion: The proposed doses of Hydroxychloroquine (HCQ) for treatment of COVID-19 (800 mg first day then $400 \mathrm{mg} /$ day for 5 days) in many guidelines worldwide are considerably higher than the maximum recommended daily safe doses ( $\mathrm{HCQ} ; \leq 5.0$ 
$\mathrm{mg} / \mathrm{kg} /$ day) for development of retinal toxicity. Also, irreversible retinal damage can occur if the exposure to the safe doses is $>5$ years. The prevalence of retinal toxicity (Bull's eye maculopathy) due to $\mathrm{HCQ}$ is $7.5 \%$ with long-term use of the drug. This was unusual in the context of HCQ causing reversible visual damage that too when taken for a short duration for 5 days. The major limitation of the case was the lack of ophthalmic examination because of the lock down effects.

Conclusion: Routine baseline ocular examination is not absolutely necessary for patients with COVID-19 who are undergoing treatment with $\mathrm{HCQ}$ but should be considered if manpower and expertise are available. It is relevant, however, to take a history of ocular disease, particularly macular disease, in patients with COVID-19 who are older than 50 years before prescribing $\mathrm{HCQ}$ as treatment, to rule out age-related macular degeneration.

Keywords: Hydroxychloroquine (HCQ), Bull's eye maculopathy, COVID-19

Conflict of interests: No comments.

FIRMST20_AB_67

Non-Clinical Sciences - Physiology

“THE EFFECT OF LEFT NOSTRIL BREATHING (LNB) AND RIGHT NOSTRIL BREATHING (RNB) ON

CARDIOVASCULAR PARAMETERS": A SYSTEMATIC REVIEW.

\section{Sheela BARGAL1, Vivek NALGIRKAR1}

1DR. D.Y. Patil medical college, Nerul Navi Mumbai, India.

Background: Hypertension is an important and growing public health challenge worldwide. If we believe in the principle of old is gold, then yoga is most effective and widely believe to reduce Blood pressure (BP) and pulse rate (PR). Hypertension has been reported to be generally associated with sympathetic overactivity. It is found that mean pulse rate /min, systolic blood pressure (SBP) and diastolic blood pressure (DBP) decreases significantly just after 5 minutes of Left nostril breathing (LNB) exercise. In the yogic system of breathing the left nostril dominance correspond to 'ida' svara with parasympathetic activation and right nostril corresponds to 'Pingala Nadi' with sympathetic activation.

Aims \& Objectives: The present study is to evaluate the effect of left nostril breathing(LNB) \& right nostril breathing (RNB) on cardiovascular parameter.

Material \& Method: This paper is a systematic review of primary research of LNB and RNB on cardiovascular parameters. The search included English language literature from 1997 to 2019. The following database were used: pubmed, web of science, Google scholar, and manual search as a source, using following terms: Left nostril breathing, Right nostril breathing, ANB, UNB, different type of pranayama. A separate search was conducted for each of these terms. This review includes 18 randomized control studies. The following inclusion criteria were used: primary research; on LNB, RNB, ANB. Studies were excluded: if not primary research; if $L N B, R N B$ was not the sole intervention and if results were reported of multiple interventions instead only of LNB, RNB. This review paper aimed to collect published primary scientific evidence on the benefits of this specific yogic breathing technique, so studies involving only ANB is not includes in the review paper.

Result: Therefore, this review showed pranayama breathing through right nostril (RNB) results in increased sympathetic activity were as left nostril breathing (LNB) reduced it. A systematic review has been conducted on various studies in pubmed and manual search as a source.

Conclusion: This review showed that LNB and RNB exercise have effect on cardiovascular parameter in normal healthy young human being. The review also emphasizes on future thrust in area of research on effect of breathing exercise LNB, RNB on cardiovascular parameters.

Keywords: Yogic breathing, LNB, RNB, SBP, DBP, pranayama.

Conflict of interests: No comments. 


\section{FÎ́RMST}

4-5 Aug 2020 | Moscow

FIRMST20_AB 64

\section{A QUESTIONNAIRE BASED STUDY REGARDING THE KNOWLEDGE, ATTITUDE AND PRACTICE OF SELF- MEDICATION AMONG UNDERGRADUATE MEDICAL STUDENTS. \\ Pradnya DEOLEKAR1, Pramila YADAV1, Simran BHATIA1 \\ 1Dy Patil School of Medicine, Mumbai India.}

Introduction: The term self- medication implies taking medicines to treat one's self without supervision of a health care personnel. Even though there are major risks in self- medication, it is a common practice seen among the general public. However this practice has a greater importance to medical students since they have the knowledge about diseases and drugs.

Materials and Methods: A cross-sectional study was conducted among 4002 nd year medical students at a medical college in Nerul, Navi Mumbai. A questionnaire about self-medication practice was distributed to students. Data was interpreted in counts and percentages.

Results: $325(81.25 \%)$ students took self-medication. The remaining $75(18.75 \%)$ students did not take selfmedication. The most common causes of selfmedication was headache 258 (79\%), cough, cold, sore throat, 250(76.9\%), fever 200 (61\%), stomach-ache $168(51.6 \%)$ and diarrhea $152(46.7 \%)$. 55(16.9\%) took self- medication before exams. Analgesics (80\%) were the most common drugs used for self-medication followed by multivitamins (61\%), decongestants $(60 \%)$, antibiotics $(46 \%)$, antipyretics $(42 \%)$ and antispasmodics (40\%). The remaining drugs used were antacids, modafinil and beta blockers which were $24.6 \%, 21.5 \%, 16.9 \%$ respectively.

Conclusion: Self-medication is wildly popular among medical students. Self-medication is both beneficial and hazardous. Hence it is important to educate medical students about the pros and cons of selfmedicating as they are the future health professionals.

Keywords: Self-medication, Medical students.

Conflict of interests: None
FIRMST20_AB_62

Allied Sciences - Cost/Economic Issues in Healthcare

\section{COST EFFECTIVENESS OF ANTIHYPERTENSIVE DRUGS} AVAILABLE IN INDIAN MARKET.

\section{Pramila YADAV1, Pradnya DEOLKAR1, Ishita}

\section{Chauhan 1}

1D.Y Patil University, school of medicine, Navi Mumbai, India.

Objective: Rising cost of medical therapy is a major concern for patients, and there is high variability in the prices of numerous branded medicines available in India. thus, cost analysis study was carried out to assess the brand price variations in the oral antihypertensive drugs available either singly or in combination and number of manufacturing companies for each, also to evaluate the difference in cost of different brands of same active drug by calculating percentage variation of cost.

Materials and methods: The cost of different brands of commonly used antihypertensive drugs was sorted out by referring latest "Current Index Of Medical Specialties" October- December, "Indian Drug Review", monthly index of Medical specialties December. The cost 10 dosage forms (tablet/ capsule) in INR of each brand, cost ratio and percentage cost variation were calculated.

Result: The percentage variation in the cost was above $100 \%$ with most of commonly used antihypertensive drugs like Amlodipine (5mg): 864.70\%, Atenolol (25mg):412.33\%, Telmisartan (40mg):277.38\% and Enalapril (10mg): $520.08 \%$ and among the combination therapy, Amlodipine + atenolol $(5+50 \mathrm{mg}): 490.37 \%$, Amlodipine + losartan $(5+50 \mathrm{mg}): 440 \%$, Atenolol + hydrochlorothiazide (50+12.5mg):504.2\%, Telmisartan+ Hydrochlorothiazide $(80+12.5 \mathrm{mg}): 401.67 \%$.

Conclusion: Despite the implementation of price control, brand price variation still exists widely for 
commonly used drugs. Re-assessment of pricing policy and implementation of quality norms is needed.

Keywords: Pharmacoeconomics, brands, price control, price variation.

Conflict of interests: None

FIRMST20_AB_61

Non-Clinical Sciences - Pharmacology

ASSESSMENT OF KNOWLEDGE OF 2ND YEAR MEDICAL STUDENTS REGARDING PROMOTIONAL DRUG

LITERATURE USING THE WORLD HEALTH

ORGANISATION CRITERIA.

Pramila YADAV1, Disha CHAUDHARY1, Pradnya

DEOLEKAR1

1DY Patil School of Medicine, Mumbai,India.

Introduction: Education of medical students has a crucial role to play in preparing future practitioners to respond appropriately to drug promotion. Inappropriate prescribing practices without necessarily benefiting the patients contribute to increased health care costs if prescriber is not aware of the World Health Organisation(WHO) criteria for ethical medical drug promotion 1988. Hence, this study was conducted with objective to assess the knowledge of 2 nd year medical students about WHO criteria for promotional drug literature.

Materials and methods: This observational study was conducted in the Pharmacology department of a tertiary care hospital. 200 2nd year medical students were given drug promotional literature and were asked to evaluate it according to the WHO criteria on prescribed pro forma.

Results: $41 \%$ of medical students were aware of the WHO criteria. $11 \%$ calculated the importance of reference of scientific literature. 35\% evaluated the importance of name and address of manufacturer or distributor. $30 \%$ evaluated necessity of dosage form and regimen. $72 \%$ evaluated the importance of active ingredients. $45 \%$ students evaluated the importance of other ingredients or adjuvant known to cause problem. $85 \%$ students calculated the importance of major drug interactions. $74 \%, 78 \%$, and $62 \%$ students calculated the importance of drug related precautions, contraindications, and warnings. $100 \%$ students calculated the importance of brand and generic name and approved therapeutic uses by $98 \%$ students.

Conclusion: The study showed that medical students have less knowledge about the WHO criteria of promotional drug literature. They require constant education regarding the WHO guidelines as these literatures often influence prescribing behaviors of physicians.

Keywords: 2nd Year MBBS students, Promotional literature, WHO criteria.

Conflict of interests: None

FIRMST20_AB_60

Research Methodology \& Implementation - Basic Research

\section{OPINIONS OF MEDICAL TEACHERS REGARDING}

ONLINE LEARNING: A QUESTIONNAIRE STUDY.

Pramila YADAV1, Ravleen K SURI1, Pradnya

DEOLEKAR1

1DY Patil UNIVERSITY school of medicine, Delhi, India.

Introduction: The COVID-19 pandemic has negatively impacted the routine teaching worldwide as a result of lockdown measures being taken for prevention. As the Education system has adapted to online teaching, it will be important to assess the changes being introduced in response to this pandemic's impact on education. During this pandemic, many teachers are required to hone their online teaching skills to delve into this new digital classroom era. This study was conducted to assess change in the Classical Learning and highlight the issues with Online Learning in India. 
Materials and Methods: This is a cross-sectional survey with a validated questionnaire was distributed using online platforms to Medical teachers, and then the results were analyzed into statistical data.

RESULT: Feedback received from 74 responses: $9.5 \%$ preclinical, 20.3\% Para clinical, 37.8\% clinical.40.5\% teachers assessed student's performance by online mcq quiz. More than half preferred 0.5-1 hr. breaks. $62.2 \%$ possess proper knowledge of technology required to conduct online lectures. When compared to classroom teaching in terms of interactivity and problem solving, around 33 to 36 stated satisfactory.

When compared to classroom teaching in terms of Practical demonstration, 30 stated poor, and 26 stated satisfactory.60\% teachers take online lectures on zoom. 65.3\%teachers provide supplementary material by presentation slides. $42.7 \%$ alleged no financial setback due to online teaching.

Conclusion: This covid 19 pandemic has made the entire teaching industry adopt the new online teaching methodology with little or no preparation in hand. This study gives new understanding of the educational scenario, analyzing the factors affecting blended learning and recommend suggestions so that education can have a smooth transition towards online learning in those areas without much hassle.

Keywords: COVID-19, pandemic, teacher, medical , Assessment, classroom , learning, teaching

Conflict of interests: None

FIRMST20_AB_58

Clinical Sciences - Cardiology

COST EFFECTIVENESS ANALYSIS OF ORAL ANTIPLATELET DRUGS.

Pradnya DEOLEKAR1, Pramila YADAV1, Praneet BEDI1 1D Y Patil school of medicine, Navi Mumbai, India.
Background: Cardiovascular disease is both highly prevalent and exceedingly costly to treat. Several novel antiplatelet agents have been found to be effective in reducing the morbidity and mortality associated with cardiovascular disease. There is a wide variation in the prices of oral antiplatelet drugs marketed in India. Thus, a study was planned to find out the variation in cost in the oral antiplatelet drugs available in India either as a single drug or in combination and to evaluate the difference in cost of various brands of the same antiplatelet drugs by calculating percentage variation in cost.

Methods: The cost of different brands of commonly used oral antiplatelet drugs was sorted out by referring latest "Current Index of Medical Specialties" , "Indian Drug Review" (IDR) The cost of 10 dosage forms (Tablets / capsules) in INR of each brand, Cost Ratio and Percentage Cost Variation were calculated. RESULTS: The percentage variation in cost for oral antiplatelet drugs marketed in India was found to be Clopidogrel 75mg (692.75\%), Dipyridamole 100mg (299.59 \%), Dipyridamole 25 mg (292.01 \% ), Clopidogrel 150mg (263.26\%), Aspirin 50mg (137.56\%), Aspirin150mg (125.71\%),Aspirin75mg(71.05\%), Prasugrel10mg (61.29\%), Clopidogrel 300mg(57.89\%), Ticlopidine $250 \mathrm{mg}$ (56\%), Prasugrel 5mg (28.57\%). The lowest percent cost variation found is ticagrelor $90 \mathrm{mg}(0 \%)$. In combination therapy the highest price variation (508.32\%) was found for Aspirin (150mg) + Clopidogrel (75mg) followed by Aspirin (75mg) + Clopidogrel (75mg) (205.88\%).

Conclusion: Despite the implementation of price control, brand price variations still exist widely for commonly used drugs. Re- assessment of pricing policy and implementation of quality norms is needed.

Keywords: Antiplatelet drugs, Cost ratio, Price variation.

Conflict of interest: None 


\section{FÎ́RMST}

4-5 Aug 2020 | Moscow

FIRMST20_AB_57

Clinical Sciences - Dentistry

INTEGRATING MULTIDISCIPLINARY APPROACH WITH ADVANCED TECHNIQUES IN DIAGNOSING

DILEMMATIC BONE PATHOLOGIES.

Harpreet Kaur ARORA1, Deepika MISHRA1, Sunny

KALA1, Rahul YADAV1

$1 A l l$ India Institute of Medical Sciences, Delhi, India.

Introduction: Histopathology is considered as gold standard for diagnosis. However, various bone pathologies such as fibro-osseous lesions (FOLs) are diverse spectrum of lesions exhibiting considerable degree of overlap with malignant tumors [such as low grade osteosarcoma (OS)]. The confluence of pathology with clinical and imaging details forms the cornerstone to decide correct set of differentials. As FOLs are the result of heterogeneous genetic mutations resulting in overexpression of certain proteins. Thus, advanced diagnostic modalities such as immunohistochemistry [detect protein expression] and molecular techniques [detect genetic mutations] are valuable adjuncts for final diagnosis. This study was planned to evaluate adjunctive specific immunohistochemical marker and genetic mutations in accurate characterisation of benign FOLs and their distinction from OS.

Material and methods: This was a hospital based retrospective study carried out in department of Oral Pathology AIIMS, New Delhi. Total 78 human biopsy samples including osteosarcoma and benign mimics were included in study group. The immunohistochemical staining was performed with MDM2, CDK4, parafibromin, BCL-2 and Gal-1 for each sample respectively.

Results: MDM2 showed $100 \%$ specificity for diagnosing osteosarcoma. CDK4 and Gal-1 showed linear increase in immunoexpression from BFOLs to OS with sensitivity of $50 \%$ and $63 \%$. BCL-2 showed equivocal immunopositivity in $O F$ and $O S$. Parafibromin showed highest immunoexpression in FD followed by OF and OS with sensitivity and specificity of $48 \%$ and $43.2 \%$ respectively.

Conclusion: Combination of Gal-1 [highest sensitivity] and MDM2 [highest specificity] can be used as an adjunct to multidisciplinary approach in accurate characterisation of these lesions.

Keywords: Fibro-osseous lesions, FOLs, MDM2, Galectin, Osteosarcoma

Conflict of interests: No conflict of interest.

\author{
FIRMST20_AB_56 \\ Clinical Sciences - Neurology
}

MICROWAVE ENCEPHALOGRAPHY (MWENC) PROJECT - NEW TECHNOLOGY OF THE BRAIN DIAGNOSTIC.

Leonid BRUSILOVSKY1, Polina BRUSILOVSKAIA1

1 NeuroVita Clinic, Toronto, Canada.

Our experimental studies have confirmed brand new information -switching theory of the human brain found a human brain bio-field in the range from 1.5 to $5.0 \mathrm{GHz}$ with a signal power of $-120 . .-100 \mathrm{dBm}$. Based on these experimental studies, a project was proposed for the development of a real-time MVEG microwave encephalography technology. Microwave encephalography MWEG is designed for early diagnosis of organic and mental diseases of the human brain. MVEG technology will provide physicians with a fundamentally new objective channel for diagnosing the mental and physiological state of the brain by recording and analyzing their own microwave radiations. Microwave encephalography MWEG will find its application in psychiatry for the diagnosis of aggression, depression and psychosis, in militarymedical, medico-social, clinical-social and labor expertise, in neurological and neurosurgical practice, in military medicine and medicine of catastrophes. The development of the Neuro interface using MWEH technology will open up broad prospects for the creation of a whole range of equipment for wheelchair users, for the visually impaired and hearing impaired (blind, deaf). The MVEG project is designed for 4 years, including the development, production of working documentation, the production of a pilot lot and the preparation of serial production.

Keywords: Encephalography, brain, diagnostic, psychiatry, mental health.

Conflict of interests: No conflict of interest. 
FIRMST20_AB_55

Clinical Sciences - Surgery

\section{EFFICIENCY OF SANITATION WITH OZONIZED}

SOLUTION AFTER THE LIVER ECHINOCOCCECTOMY.

Rasul Abakarovich KOICHUEV1, Lloyd Tadiwa

KASHANGURA1, Khalid KOICHUEV1

1Dagestan State medical university, Makhachkala, Russian Federation

Introduction: The search for more effective and less traumatic methods of treating liver echinococcosis (EN), development of surgical approaches depending on location of cysts and nature of lesion; relapse prevention; methods improvement for eliminating residual cavities; solving the problems of hemo- and cholestasis, reducing frequency of postoperative infectious and inflammatory complications. Aim was selecting optimal tactics for treatment of liver echinococcosis by clinical and experimental development, implementing method of using medicinal ozone for residual cavities (RC) treatment after echinococcectomy .

Materials and Methods: Study included patients who underwent organ-preserving surgeries for liver echinococcosis at Clinical Hospital of Makhachkala. Sampling was formed from patients in study. Had 233 patients who underwent surgical interventions for echinococcal cysts, sizes which exceeded $12 \mathrm{~cm}$. Each surgical intervention was completed with drainage of RC. Echinococcectomy of liver with external drainage of RC without reducing volume was performed in 117 patients in sample. Assessment of the RC rehabilitation various methods effectiveness, dynamics of their reduction was carried out.

Research results: Episodes of state deterioration, expressed in appearance of pain in liver, increase in body temperature, sweating and appearance of leukocytosis in immediate postoperative period, were observed with decrease in volume of discharge from drainage. Washing residual cavity with antiseptics within 3 days, number of patients in first group, where seeding didn't give growth, reached 8 (72.7\%), in second group, seeding did not give growth only in 4 ( $25.0 \%)$ patients $(\chi 2=4.24 \mathrm{p}=0.039)$. minimum volume of cavity, possibility of cutting sutures didn't allow introduction of sufficient amount of antiseptic solution under dosed pressure. possibility of due lavage of RC with antiseptic solutions in presence of drainage, and introduction of the method developed by us, based on use of medical ozone for its sanitation, ensures prevention of development of purulent complications of echinococcectomy.

Conclusion: effectiveness of sanitation with ozonated solution after echinococcectomy showed improvement in results, had an antihypoxic effect, manifested sorption properties, stimulated micro- and macrophage system of liver, proving high efficiency of using ozone for disinfecting germ elements of cystic echinococcus, preventing suppuration of residual cavities.

Keywords: Echinococcectomy, residual cavity, fibrous capsule, ozone

Conflict of interests: No conflict of interest.

\author{
FIRMST20_AB_54 \\ Clinical Sciences - Surgery
}

THE CYTOLOGICAL CHARACTERISTIC OF PERITONEAL FLUID AGAINST THE BACKGROUND OF INTRODUCTION OF OZONIZED PHYSIOLOGICAL SOLUTION IN EXPERIMENT

Ragimov Razin MIRZEKERIMOVICH1, Abdullaeva

Naida MURTAZALIEVNA1,

Dorcas AWUNI1, Suraj KUMAR1

1Dagestan State Medical University, Makhachkala, Russian Federation

Introduction: The medications injected into the peritoneal cavity are easily adsorbed by cells of the peritoneum, which contributes to their initial concentration in the bloodstream. However, the reaction of the peritoneum and it associated changes in the composition of the peritoneal fluid (PF) in response to the introduction of medical ozone, widely used in clinical practice and ozonized saline solution (OSS) has been inadequately explored.

Objective: A comparative study of cytodynamics (PF) against the background of OSS and ozone-free SS introduction. 


\section{FÎ́RMST}

Materials and Methods. The trial involved white male rats weighing $140-160 \mathrm{gr}$; the rats were divided randomly into 2 series. The 1 st series introduced OSS into the peritoneal cavity $(2 \mathrm{ml}$ per $100 \mathrm{gr}$ of body mass), the 2 nd series were given the SS. Five (5) intact rats were used as control cases. The cellular composition of the peritoneal fluid was studied on the 1st, 2nd, 3rd, 7th and 14th experimental days. Peritoneal smears were stained by RomanovskyGiemsa technique. Statistical processing was performed by the "Biostat" computer programme.

Results: Normal rats have $10-16$ cells per $8000 \mu \mathrm{m} 2$, mean $-13,33 \pm 1,83$. Approximately $31 \%$ of all these cells are presented by neutrophils, macrophages and lymphocytes with fewer monocytes, mast cells and eosinophils.Introduction of OSS promotes increase in number of cells (up to $16,44 \pm 1,65 ; p<0,05$ ), presented mainly by neutrophils. Eosinophils and mast cells are few $(M e=0, p 25=0, p 75=1, \min =0, \max =2)$, the latter being in the stage of degranulation. The number of cells elevated up to $18,22 \pm 2,52$, cellular composition being presented by neutrophils and large lymphocytes as well $(1,78 \pm 0,96 ; p<0,05)$. On day 3 , the density of PF cells reduces with no different result of the 7 th day from the control. The 2nd series showed no changes in PF cells density and their relative composition on 1st and 2 nd days, $(14,78 \pm 0,66)$, despite the presence of medium-sized $(\mathrm{Me}=2, \mathrm{p} 25=1, \mathrm{p} 75=2, \min =1, \max =3$ ) and large lymphocytes $(\mathrm{Me}=2, \mathrm{p} 25=1, \mathrm{p} 75=2, \min =0$, $\max =2$ ). On the 3rd day, cellular composition of PF does not differ from the control.

Conclusion: On the background of OSS introduction, the amount of PF within the first two days increases due to neutrophilic granulocytes $(p<0,05)$ and to a lesser extent - large lymphocytes, which might be due to response of the peritoneum to ozone.

Keywords: White rats, Ozone, Peritoneal fluid..

Conflicts of interests: None

FIRMST20_AB_53

Clinical Sciences - Surgery
INFLUENCE OF OZONIZED SALINE SOLUTION ON THE

COURSE OF PURULENT PERITONITIS IN THE EXPERIMENT: FOCUS ON THE CYTODYNAMICS OF PERITONEAL EXUDATE

Mohd Saleem MALIK1, Abduselimova Jennet

AMRULAKHOVNA1,

Ragimov Razin MIRZEKERIMOVICH1, Abdullaeva Naida MURTAZALIEVNA1

1Dagestan State Medical University, Makhachkala, Russian Federation

Aim: Important component of antimicrobial protection in peritonitis is the system of phagocytic cells. Therefore, along with lavage, stimulating this line of defense is of great importance. For this purpose, we propose an intraperitoneal infusion of ozonized saline solution, since medical ozone has not only bactericidal but also immunomodulatory effects.

Materials \& Methods: Used white male rats 3-4 months age, weight $140-160 \mathrm{~g}$. After reproducing the model of fecal peritonitis according to S.S. Remennik (1966), 1st series were injected with ozonized physiological solution into the abdominal cavity at the rate of $2 \mathrm{ml}$ per $100 \mathrm{~g}$ of mass, In the $2 \mathrm{nd}$ - physiological solution. The cell composition of peritoneal fluid was studied on 1st,2nd,3rd,7th \& 14th days of the experiment using the hardware-software complex"MEKOS - Ts1". Peritoneal swabbing were stained according to Romanovsky-Giemsa in our modification. Statistical processing was carried out using the computer version (4.03) of the program"Biostat".

Results: On days 1-3, the RR in rats of 1st series was $132 \pm 5.7 / \mathrm{min}$, tousled hairs, were inactive. By the 714 th day in surviving rats $(80 \%)$, these manifestations were normalized.

On 1st day, the no of neutrophils increased by 3 times (up to $13.56 \pm 0.87$ ) \& eosinophils $(p<0.05)$. fragments of nuclei are visible in macrophages. On 3rd day, the cell density of the exudate decreases to $16.22 \pm 1.56$. On 1st day it averaged $31.67 \pm 2.61$ on the smear area $(8000 \mu \mathrm{m} 2)$. On the 14th day, the cellular composition approaches that of in normal rats. In 2 nd series, on days 2-7, a mass death was noted (up to $56 \%$ ). On the 1 st day, no of neutrophils \& monocytes increases by 4.5 times, eosinophils and destructive cells - more than 10 times. The smears increased the fibroblasts (up to $3.22 \pm 0.66$ ) and destructive cells (up to $7.67 \pm 1.23$ ). On the 3rd-7th day, destructive cells increase sharply 


\section{FÍRMST}

4-5 Aug 2020 | Moscow

(up to $40.6 \%$ ) \& clear decrease in neutrophils. Acanthocytes \& cocci with coli in fibrin accumulations are detected in all fields.

Conclusion: Intra-abdominal administration of ozonized saline in peritonitis helps to reduce mortality and delays the spread of inflammation along the peritoneum.

Keywords: Peritonitis, Ozone, Peritoneal Exudate.

Conflicts of interests: None

FIRMST20_AB_52

Clinical Sciences - Anesthesiology \& Intensive Care

PERIOPERATIVE ASSESSMENT OF DIAPHRAGMATIC MOVEMENT BY ULTRASOUND AND ITS INTERACTION WITH POSTOPERATIVE PULMONARY COMPLICATIONS FOLLOWING UPPER ABDOMINAL SURGERIES FOR CANCER: A LONGITUDINAL OBSERVATIONAL STUDY

Prasanna Vani VANAMAIL1, Kalpana, BALAKRISHNAN1

1 Cancer Institute (W.I.A.), Adyar, Chennai, India

Aim: Postoperative pulmonary complications (PPCs) require early recognition to reduce morbidity and mortality. Diaphragmatic dysfunction is associated with upper abdominal surgery and predisposes patients to PPCs. We aim to assess the peri-operative changes in amplitude of diaphragmatic movement by ultrasound with reference to PPCs. We compared the difference in diaphragmatic inspiratory amplitude between PPC and No-PPC group and evaluated the trend in serial diaphragmatic inspiratory amplitude measurements within each group.

Methods: We conducted a prospective, observational study in patients between the ages 18 to 75 years undergoing elective, open, upper abdominal oncological surgeries under combined general and epidural anesthesia. We performed ultrasonographic evaluation of the diaphragm by measuring the diaphragmatic inspiratory amplitude in the right and left hemi-diaphragms during quiet and deep breathing both preoperatively and on postoperative days 1, 2 and 3. PPCs were diagnosed and graded based on standard criteria by an independent investigator who was blinded to the ultrasound measurements. Patients were followed-up for PPCs until day 7.
Results: One hundred and sixty-two patients were included in the final analysis, which included 33 patients in the PPC group and 129 in the No-PPC group. The preoperative diaphragmatic inspiratory amplitude was significantly less in the PPC group when compared to the No PPC group $(P<0.001)$, while there was no significant difference in the postoperative measurements, except in the left hemi-diaphragm during deep breathing, which exhibited a reduced diaphragmatic inspiratory amplitude on postoperative day 1 in the PPC group. In the No-PPC group, diaphragmatic inspiratory amplitude on postoperative day 3 was significantly higher than on day 1 in the right hemi-diaphragm during quiet breathing. Post-operative day 3 diaphragmatic inspiratory amplitude was higher than on postoperative day 2 also in the left hemi-diaphragm during deep breathing in the No-PPC group. No improvement in diaphragmatic inspiratory amplitude on day 3 was observed in the PPC group.

Conclusions: Decreased preoperative diaphragmatic inspiratory amplitude and its impaired recovery after surgery was observed in patients with PPCs. Perioperative assessments of diaphragmatic movements by point of care ultrasound helps identify patients at risk of a PPC following upper abdominal oncological surgeries.

Keywords: Postoperative pulmonary complications, diaphragm, ultrasound, upper abdominal surgery, postoperative pneumonia

\section{Conflicts of interests: None}

FIRMST20_AB_51

Clinical Sciences - Oncology \& Hematology

\section{IMMUNE EVASION OFFERS NOVEL INSIGHTS INTO THE EVOLUTIONARY TRAJECTORY OF UROLOGY'S MOST DEADLY CANCER.}

Faiz JABBAR1

$1 U C L$, London, United Kingdom

Background: Renal cell carcinoma (RCC) is the deadliest urological malignancy. Although the treatment of primary RCC has become more successful, five-year survival rates for metastatic RCC remains low at $8 \%$. Large-scale studies of metastatic disease have not analysed paired primary-metastatic tumours, a requirement to distinguish between 


\section{FÎ́RMST}

4-5 Aug 2020 | Moscow

tumours with and without metastatic potential. An improved understanding of the genetic differences between primary and metastatic tumours could reveal exploitable vulnerabilities that may prevent metastasis and/or improve prognosis.

Aim: Immune evasion is required for metastasis. We investigated whether genomic alterations causing allelic imbalance to human leukocyte antigen (AIHLA) alleles would facilitate immune evasion, subsequently promoting proliferation and metastasis.

Method and Results: We acquired the ability to decipher potential modes of metastatic progression through simultaneous analysis of 418 primary and 278 metastatic biopsies from 31 renal cell carcinoma patients. Multiple regions from each tumour were biopsied giving us clonal resolution. Al was investigated using fluorescently labelled STR oligonucleotides that were polymorphic within the HLA locus. I categorised them into three groups based on evidence of selection in the metastasis. "Selected" frequencies were compared to "not selected". Significance was calculated by comparing event selection proportions, to null background mutation rates. AlHLA was significantly associated with metastasis, suggesting AlHLA contributes to metastasis $(p=0.002)$. Immunohistochemical analysis on 897 tumour biopsies stained for the proliferation marker Ki67 showed increased proliferative potential in AIHLA-positive versus AIHLA-negative biopsies.

Discussion: These results suggest escape from immune predation represents a significant constraint to tumour evolution and AIHLA could facilitate evasion, thereby contributing to metastasis. An investigation of the HLA alleles lost may help determine which neoantigens will elicit an effective immune response and so could be exploited by personalised immunotherapies.

Keywords: Evolution, metastasis, immune evasion, renal cancer

Conflicts of interests: None

FIRMST20_AB_50

Allied Sciences - Digital Health

\section{WEARABLE ANGER MONITORING SYSTEM (WAMS).}

\section{Mohammed SAHAD1}

1N.P. Ogarev State Medical University, Republic of Mordovia Russian Federation

Anger is a natural, healthy emotion. However, it can arise out of proportion to its trigger. In these cases, the emotion can be impede a person's decision making, damage relationship and otherwise cause harm. Learning to control anger can limit the emotional damage. Thus as a tool to control anger, a wearable anger monitoring system has been developed. The system is quick, easy and discreet during the recording and retrieval of self-tracking information and provide the additional benefits of always being with the user, thus facilitating more accurate in-vivo recordings. Moreover the system gives a periodic self-assessment. The system uses relevant sensors connected to a microcontroller unit (ESP232) that extract the real time data and to determine the current emotion status via mathlab algorithm. The analysis includes data collection using the hardware developed. The device would contribute in the long term Healthcare.

Keywords: Anger monitoring system, discreet, in-vivo recordings.

Conflicts of interests: High Accuracy, Inexpensive, Can be customized, Wearable Technology, Low Power Consumption, Improving personal relationships, Increased quality of life.

FIRMST20_AB_49

Clinical Sciences - Ophthalmology 


\section{FÎ́RMST}

4-5 Aug 2020 | Moscow

LATANOPROST AND BIMATOPROST, SO SIMILAR, BUT WHICH IS BETTER?

\section{Karishma SHAH1}

1DY Patil University School of Medicine, Navi Mumbai, India.

Introduction: Both Latanoprost and Bimatoprost are Prostaglandin analogs, which are first line treatment for Primary open Angle Glaucoma (POAG), but we took a closer look into their comparative efficacy with some simple office procedures.

Aims:

1.To compare the peak and rise of Intra ocular pressure (IOP) from baseline during the modified Diurnal Tension Curve (mDTC) and Water Drinking Test (WDT) achieved using 2 different prostaglandin analogs in 2 eyes of the same patient with symmetrical damage; and thereby analyze the efficacy of both drugs in blunting the IOP spike.

2. To assess the relationship between the peak IOP by water drinking test and the peak of modified diurnal curve.

3. To establish the importance of water drinking test in the present day scenario.

Materials and methods: This was a prospective, observational and interventional study over a period of 8 months done at Aditya Jyot Eye Hospital, Mumbai.31 subjects (62 eyes) with mild-moderate bilateral symmetric Primary Open Angle Glaucoma (POAG) were started on eye drops Bimatoprost $(0.03 \%)$ in the right eye and Latanoprost $(0.005 \%)$ in the left eye and were followed up after 3 weeks for water drinking test (WDT) and modified diurnal tension curve (mDTC)

Results: At the end of our study we found that the right eye on bimatoprost $(0.03 \%)$ showed lower peak IOP's , and lower difference between peak and baseline IOP measured over the whole day (MDTC) and on provocation (WDT) compared to the left eye on latanoprost $(0.003 \%)$. We hence propose that bimatoprost may have more IOP lowering efficacy than latanoprost.The WDT showed a definite peak at 30-45 $\mathrm{min}$ in all patients that would have been missed in routine clinic single IOP recording.Both $\mathrm{MDTC}$ and WDT recorded a rise in IOP from baseline, but the difference between baseline and peak IOP's was significantly higher in WDT than in MDTC, and the peaks of both did not correspond.
Conclusion: This study corroborated previous reports that demonstrate the clinical applicability of WDT as a useful tool in assessing the status of the eye's outflow facility and determining IOP peaks that would otherwise have been missed in routine clinic IOP measurements. Furthermore prospective studies are required to evaluate the IOP peaks and fluctuations with both drugs and a greater sample size to compare their IOP lowering efficacy on a larger scale.

Keywords: Latanoprost, Bimatoprost, Primary Open Angle Glaucoma (POAG), Water Drinking Test (WDT), Intra ocular pressure (IOP).

No interests of conflicts: No financial interest/disclosure

FIRMST20_AB_47

Clinical Sciences - ENT

\section{PREVENTION OF DAMAGE TO THE RECURRENT}

LARYNGEAL NERVES DURING THYROID SURGERY, CLINICAL MANIFESTATIONS AND TREATMENT PATIENTS.

Anatoly ROMANCHISHEN1, Kristina VABALAYTE1, Filipp ROMANCHISHEN1, Tatyana GOTOVYAKHINA1 St. Petersburg State Pediatric Medical University, St. Petersburg, Russian Federation.

One of the dangerous complications after thyroid operations is one - or two-sided larynx palsy and impaired mobility of the vocal folds. The reason is the surgeon's insufficient knowledge of the topographic anatomy of the neck and mediastinum. Voice changes can be determined by trauma of larynx during intubation, exacerbation of chronic laryngitis.

Materials and methods: The study included 390 patients operated on the thyroid gland in our Center from July 2009 to July 2011 for nodular, diffuse toxic goiter, thyroid cancer T1-3 (14,6\%). Average age 52.1 \pm 9.6 ears. Men made $11.0 \%$, women $89.0 \%$. Thyroid resection, thyroidectomy, thyroidectomy with central lymphadenectomy were performed. In total, 650 thyroid lobes were removed in 390 patients. In the course of each operation, identification and dissection of RLN was performed. Both video laryngostroboscopy and telefaringolaryngoscopy (PULSAR II) were used. Patients were examined before surgery and on the 2 nd 


\section{FÎ́RMST}

4-5 Aug 2020 | Moscow

day after surgery. In a case of impaired mobility of the vocal fold $(8.6 \%)$, conservative therapy (neuromidin , milgamma, ascorbic acid, glucocorticosteroids ) and early phonopedic correction were prescribed from day 1 Within a year, the mobility of the vocal fold recovered in $87.5 \%$ of cases. Paralysis of the muscles of the larynx was noted in $1.1 \%$. The average recovery period for mobility of the larynx was $1.8 \pm 1.2$ months. Conclusion. The only possibility to avoid damaging nerves return is their visual inspection (Lahey F.H., 1938; Lennquist S., 2004) during each operation. A thorough analysis of the anatomy and topography of the RLN was undertaken at the clinic of F.A. Romanchishen in the 2000s. Pathological changes in the larynx in the early postoperative period in most cases $(72.3 \%)$ were not associated with the consequences of UHN trauma, but included: functional dysphonia (14.6\%), consequences of laryngeal trauma $(12.3 \%)$, exacerbation of signs of chronic laryngitis (5.9\%). 1757 RLN have been verified under the control of intraoperative monitoring in 1097 patients, which provided a 10-fold decrease in the frequency of postoperative paresis and paralysis of the laryngeal muscles. Videostroboscopy combined with computerized voice analysis made it possible to document the diagnosis, evaluate the effectiveness of treatment, and motivate the patient to continue the course of therapy.

Keywords: Thyroid surgery, recurrent laryngeal nerve, larynx palsy

Conflicts of interests: None.

FIRMST20_AB_46

Clinical Sciences - Urology \& Nephrology

\section{POSSIBILITIES OF SPERMATOGENESIS STIMULATION}

WITH LOW-LEVEL LASER THERAPY IN RED SPECTRUM IN MALE SECRETORY INFERTILITY.

Maria POTAPOVA1, Sergei BOROVETS1, Salman ALSHUKRII

1Academician I.P. Pavlov First Saint-Petersburg State Medical University of the Ministry of Healthcare of the
Russian Federation, Saint-Petersburg, Russian Federation

Introduction: Nowadays infertility is a rising problem worldwide. About $30-50 \%$ of infertility is related to male factor. Due to insufficient efficacy of medical therapy of secretory male infertility there is a need to develop and apply more resultative methods of spermatogenesis stimulation.

The aim of our study was to assess the efficacy of lowlevel laser therapy (LLLT) in red spectrum on sperm parameters, sperm DNA fragmentation (SDNAF) and MAR-test in men with secretory infertility.

Patients and methods. The study included 85 men with secretory infertility; 50 of them (first group) were treated with LLLT and 35 (second group) didn't have course of LLLT. Average age of patients of the first and second groups was $31.4 \pm 1.3$ and $32.3 \pm 1.4$ years.

LLLT was performed in red spectrum on a Rubin-C device (Russia). 10 laser therapy procedures were carried out every two days with active luminescence for 10 minutes of both testicles. Male hormone panel, semen analysis, MAR-test, SDNAF were assessed before and after the treatment. The normative indicator of SDNAF was considered as $\leq 15 \%$, and $\leq$ $10 \%$ was for the MAR test. The obtained data was statistically analyzed.

Results. After the course of LLLT in patients of the first group significantly increased: ejaculate volume, sperm viability, number of morphologically normal sperm forms - by an average of 11\%, $9 \%$ and $23 \%$ respectively, and active mobile sperm forms - by an average of $14 \%$ and $19 \%$ one and two months after the therapy respectively. In 12 patients of the first group with initially increased SDNAF its level became normal after the treatment. We searched out in patients of the first group significant decrease of MAR-test with its initial level less than $30 \%$. In this group we noticed increase in concentration of total and free testosterone in blood plasma within one month after the LLLT. As a result of the treatment, pregnancies developed in $10(20 \%)$ out of 50 couples.

In the second group of patients there were no any significant changes in sperm parameters and hormone concentration in blood plasma during two months of follow-up; pregnancies didn't develop.

Conclusions. 1. LLLT in red spectrum leads to significant improvement of sperm quality in patients 


\section{FÎ́RMST}

4-5 Aug 2020 | Moscow

with idiopathic secretory infertility, that contributes to spontaneous pregnancies in couples. 2. LLLT in red spectrum normalizes sperm DNA-fragmentation level. 3. LLLT in red spectrum decreases MAR-test level, if it was initially less than $30 \%$.

Keywords: male infertility; low-level laser therapy; red spectrum

Conflict of Interests: None

FIRMST20_AB_45

Non-Clinical Sciences - Public Health

\section{QUALITY OF LIFE AND BURNOUT IN PEDIATRIC DENTISTS: NO CAUSE FOR ALARM? \\ Pavel IONOV1}

1I. P. PAVLOV FIRST SAINT PETERSBURG STATE MEDICAL UNIVERSITY, MINISTRY OF HEALTH OF RUSSIA, St.Petersburg, Russian Federation.

Pediatric dentists are considered to be the group of specialists most exposed to occupational stress. Their health status, quality of life (QoL) and working conditions may have impact on quality of their working activities. During the last decade burnout syndrome has been increasingly reported among dentists. It may lead to QoL impairment and negative changes in health. By now data about QoL and burnout syndrome in pediatric dentists is lacking.

Aim: To study quality of life and professional burnout among pediatric dentists working in municipal dental clinics of St. Petersburg.One hundred and twenty dentists from 8 municipal dental clinics of St. Petersburg that provide dental care for children were included in the study. All the dentists completed generic QoL questionnaire RAND SF-36, Maslach Burnout Inventory (MBI), and checklist consisting of 10 questions regarding demographic characteristics. Emotional exhaustion, depersonalization and personal accomplishment were evaluated by $\mathrm{MBI}$; the final scores were categorized as high, moderate and low based on standard test scores. Data were analyzed using ANOVA and chi-squared test.

$93 \%$ of participants were female, mean age -47.2 years \pm 11.3 , the mean work experience -19 years. According to specialization the distribution was as follows: pediatric dentists $-n=83$, orthodontic dentists $-n=25$, dental surgeons $-n=12$. The above subgroups did not differ by age and gender $(p>0.05)$. Social, emotional functioning, mental health and vitality in dentists working in municipal dental clinics were lower than in control group $(p<0,05)$. No significant differences between subgroups of dentists according to specialization were found, except lower physical functioning of dental surgeons $(p<0,05)$. Most dentists experienced professional burnout. High degree of professional burnout was found in $7,5 \%$ of pediatric dentists according to all three scales; $62,5 \%$ had high degree of emotional exhaustion and depersonalisation with an average or low level of reduction of professional achievements; $10 \%$ had high degree of burnout according to one scale; $20 \%$ - no burnout. Significant differences were found between groups of orthodontists, dental therapists and dental surgeons. The level of emotional exhaustion and depersonalisation was significantly higher in dental surgeons and in compared with dental therapists orthodontists $(p<0.05)$

Pediatric dentists working in municipal dental clinics exhibit reduced social and mental well-being and vitality level. The majority of pediatric dentists working in municipal dental clinics reported symptoms related to burnout syndrome. The data obtained emphasize the necessity for prophylactic measures of burnout syndrome and QoL maintenance among pediatric dentists.

Keywords: Occupational stress, quality of life, burnout syndrome, pediatric dentist, orthodontic dentist, dental surgeon.

Conflicts of Interests: Occupational stress, quality of life, burnout syndrome, pediatric dentist, orthodontic dentist, dental surgeon.

FIRMST20_AB_44

Clinical Sciences - Transplantology

\section{PARADIGMS OF LIVER TRANSPLANTATION FOR} HEPATOCELLULAR CARCINOMA IN THE REPUBLIC OF BELARUS.

Denis EFIMOV1, Aliaksei SHCHERBA1, Sergey

KOROTKOV1, Oleg RUMMO1,

1Minsk Scientific and Practical Center of Surgery, Transplantology and Hematology, Minsk, Belarus.

Introduction: In spite of the number of medical technologies developed in the world for the treatment of hepatocellular carcinoma (HCC), the most acute 


\section{FÎ́RMST}

4-5 Aug 2020 | Moscow

question at the moment is the selection criteria for such for liver transplantation, the place and time of application of other methods of treatment to achieve optimal long-term results.

The aim: To evaluate the effectiveness of the liver transplantation of patients with HCC and cirrhosis according to morphological (Milan criteria) and oncological criteria.

Materials: A retrospective cohort study of patients undergoing liver transplantation from 2008 to 2019 (a total of 690 transplantations, 105 (15\%) of recipients with HCC) in the Minsk Scientific and Practical Center for Surgery, Transplantology and Hematology (Minsk, Republic of Belarus) was performed. The patients were divided into 3 groups: recipients whose liver transplantation was performed according to the Milan criteria ("Milan"), recipients that did not meet neither the Milan criteria nor the UCSF criteria ("extra-UCSF") and recipients that did not meet the Milan and UCSF criteria that meet the BCLC-B criterion and undergo locoregional therapy (LRT) with subsequent assessment of the radiological and serological response ("Lerut"). In each group, the frequency of progression in the waiting list and the recurrence rate of HCC after transplantation, hospital mortality, the frequency of arterial and biliary complications, and cancer-associated mortality were assessed.

Results: The highest rate of HCC progression on the waiting list was observed in the extra-UCSF group of patients (36\% versus $11 \%(p=0.03)$ and $15 \%(p=0.1)$ in the "Milan" and "Lerut" groups, respectively). Statistically significant differences were found in the level of cancer-associated mortality and median disease-free survival, with the worst rates in the extraUCSF group. The one-year, three-year and five-year overall survival rate in the groups were $87.5 \%$; $80.1 \%$ and $70.3 \%$ for the Milan group; $78.6 \% ; 62.9 \%$ and $62.9 \%$ for the extra-UCSF group and $96.4 \% ; 86.4 \%$ and $78.7 \%$ for the Lerut group, respectively.

Conclusion: The oncological prognosis of patients with $\mathrm{HCC}$ is formed not only from the size and number of tumor foci as from the biological characteristics of the tumor. LRT combines both a therapeutic option, that allows reach better survival, and a stratification option, that enable patients to wait for liver transplantation beyond the accepted criteria.
Keywords: liver transplantation, hepatocellular carcinoma.

Conflict of Interests/Comments: Nothing to declare.

\section{FIRMST20_AB_43}

Non-Clinical Sciences - Pharmacology.

\section{THE OTHER SIDE OF PERSONAL PROTECTIVE}

EQUIPMENTS (PPES) : A QUESTIONNAIRE BASE STUDY AMONG HEALTH CARE WORKERS WORKING IN COVID SETUP.

Prasad KAKADE1, Prasad KAKADE1, Kavitha VIVEK1, Vaishali THAKARE1

1DY Patil University School of Medicine, Navi Mumbai, India.

Title: The Other Side of Personal Protective Equipment (PPEs). A Questionnaire Base Study Among Health Care Workers Working in Covid Setup

Content: Use of PPE kits have become more helpful in the time of COVID-19 Pandemic. Health care workers and doctors are at forefront and are treating COVID-19 patients with the help of use of PPE Kits as protectives Keeping this in mind the following study was conducted with the aim to analyze the problems associated with PPEs by studying various parameters like hours of use and Hazards like Dehydration \& infections and to study the protection offered by PPEs in health care workers.

Methods: A cross sectional questionnaire based study was conducted among Health Care Workers and Doctors those who are working in COVID-19 set up after taking ethical clearance from the Institutional Review Board.

Result: A total of 102 candidates participated in this study, out of which $76.70 \%$ of the participants were working in Tertiary Care Teaching Hospital.78.1\%participants were of opinion that they use N95/FFP2 Face mask, Disposable gloves, Eye protection goggles, Cover all and Gowns, Shoe covers and Head covers as a form of PPE used in routine care of COVID-19 patients.48 \%of them faced dryness, Scales, and redness, device related pressure injuries as skin related adverse effects with the use of PPE 
Conclusion: Though PPE Kits are being used by health care workers and doctors while working in COVID-19 setup, they are facing many problems and difficulties in carrying out there working activities. So lot of research and improvement in PPE Kits is still needed.

Keywords: Personal Protective Equipments, Adverse effects, COVID-19 pandemic.

Conflict of Interests/Comments: None declared.

FIRMST20_AB_42

Non-Clinical Sciences - Public Health

\section{DIETARY HABITS OF YOUNG PEOPLE IN STRESSFUL CONDITIONS DURING A PANDEMIC.}

Aleksandra LYUDININA1, Zhanna IVANKOVA2

1IPhys FRC Komi SC UB RAS, Syktyvkar, Russian Federation.

2Pitirim Sorokin Syktyvkar State University, Syktyvkar, Russian Federation.

Introduction: The preservation and development of students' health is important. It has been reported that about $30 \%$ of students experience levels of stress and depression [1,2,3]. Symptoms of depression and perceived stress also include excessive consumption of unhealthy food, carbohydrates and overeating $[3,4]$. Recently, researchers have been studying possible associations between depression and polyunsaturated fatty acids (PUFAs), which may modify depression symptoms $[4,5]$. Based on the recommendations of several observational, epidemiological, and clinical studies, psychiatric disorders such as depression may be modified or amended by the intake of essential fatty acids (FAs) $[4,6]$. The AIM of the work was to assess of level PUFAs in diet among students and it links with the prevalence of both depression and stress.

Subjects and Methods: Our case-control pilot study included 96 young people from Russia $(n=53)$, India $(n=22)$ and Nepal $(n=21)$ (69\% female and 31\% male) (average age $19.9 \pm 2.8$ years). The study included of Russian medical students, Indian medical and engineering and Nepalese agriculture and medical ones. Self-administered questionnaires included QFat by Web resource developed by IPhys FRC Komi SC UB RAS [7], Cohen's Perceived Stress Scale and a modified Beck Depression Index [2,8]. For statistical processing, the nonparametric Kruskall-Wallis test and Spearman's correlation coefficient were used (STATISTICA 8.0).

Results: In pandemic conditions of a strict regime of self-isolation and exam session, we found the strong stress level in young people of India and Nepal are quite higher compare of Russian students - 73\%, 95\%, $6 \%$ respectively. Depressive state (moderate, severe and difficult forms) are $32 \%, 29 \%, 36 \%$ of the interviewed students from Russia, Nepal and India.The study showed a non-optimal balance of FAs in the diet of majority students, characterized by an excess of saturated and $n-6$ PUFAs in the diet against the background of insufficient intake of EPA+DHA in the body. Less optimal balance of FAs was noted among Russian students, which expressed by a high level of ratio of $n-6 / n-3(17,4 \pm 14,4 ; p=0.000)$.

Conclusion: We concluded that the part of interviewed students with moderate to difficult depression ranged from 29 to $36 \%$. The highest stress levels were among young people in Nepal and India. We found an inverse association between n-3 PUFAs intake and depression somatic symptoms only in Russian medical students.

Keywords: Pandemic, essential fatty acids, depression, stress, nutrition, PUFAs.

Conflict Of Interests/Comments: No potential conflict of interest.

FIRMST20_AB_41

Clinical Sciences - Nutrition

WEB SERVICE “QFat” FOR CALCULATION INTAKE OF ESSENTIAL FATTY ACIDS: DEVELOPMENT AND APPLICATION.

Aleksandra LYUDININA1, Ekaterina BUSHMANOVA1 1IPhys FRC Komi SC UB RAS, Syktyvkar, Russian Federation. 


\section{FÎ́RMST}

4-5 Aug 2020 | Moscow

Introduction: High incidence rate related to unbalanced nutrition is a severe problem all over the world. Developments in nutritional are an applied task of modern medicine that creation of a simple and visual method to estimate nutrition is a hot topic. Traditional dietary methods are food frequency questionnaire (FFQ) and 24-hour recalls [1]. Important to bear in mind not only it, but content and quality of fatty acids (FA) have different effects on the human body $[1,2,3]$. The AIM of the research is to create a web service (QFat) - for the quantitative and qualitative calculation of dietary fats.

Materials \& Methods: We examined 23 young people from 16 to 29 years old. QFat development consisted of few steps: 1) definition of leading fat-containing foods; 2) studying FA profile of the products; 3) digital visualization of products for more accurate weight; 4) compiling a base of norms for the FAs consumption, creating a computer program and a web service.

Results: QFat is an online questionnaire developed by our own (certificate GR № 2016662728 from 20.12.2016) and it includes an assessment of FFQ of fat-containing products and 24-hour recalls. An analogue of the QFat was n-3 PUFAs FFQ which was developed and approved to estimate consumption of n-3 PUFAs [4]. Moreover, we used FFQ presented by AMAP (№ 29-2009). Briefly, the QFat asks about intake food, including dairy products, cheeses, vegetable oils, fat products, meat, fish, sweets, nuts and fast food. Participants recorded all food consumed during one month and indicated serving sizes by using pictures.

Since level of PUFAs in the blood depends on nutrition [5], we studied profile of FAs in plasma to assess the objectivity of received data. Fasting blood sampling was performed on the same day with QFat. Synchronous trends were obtained in the profile of essential fats in plasma and in the diet. In addition, a Spearmen correlation was found between the content of $\omega-3$ EPA and DHA in the blood and in the diet. Now QFat is used in scientific research and exposition. Results obtained about association consumption of essential n-3 PUFAs with Heart Rate Variability [6] and psychophysiological status [7].

Conclusion: As far as we know, there are no analogues of this WEB service (assessment of frequency and 24hour recalls together). QFat is noninvasive, economy, simple, informative and available resource for private using.
Keywords: Nutrition, dietary fat, essential PUFAs, development.

Conflict of Interests/Comments: No potential conflict of interest.

FIRMST20_AB_40

Non-Clinical Sciences - Pharmacology

KNOWLEDGE, ATTITUDE AND BEHAVIOR OF HEALTH

CARE PROFESSIONALS TOWARDS DRUG

PROMOTIONAL LITERATURE DISTRIBUTED BY

PHARMACEUTICAL COMPANIES IN A TERTIARY CARE

TEACHING HOSPITAL.

Shruti BHAKARE1, Kavitha DONGERKERY1, Vaishali

THAKARE1, Deepak LANGADE1

1DY Patil University School of Medicine, Navi Mumbai, India.

Background: In the age of aggressive marketing of pharmaceutical products, drug promotional literature plays a crucial role. W.H.O defines drug promotion as "all information and persuasive activities of manufacturers and distributors, the effect of which to induce prescription, supply and purchase and/use of medical drugs" .Pharmaceutical companies use drug promotional literature as major marketing tool to promote their new drugs claimed to provide vital drug information. The purpose of this study was to assess physician perspective regarding pharmaceutical promotional literature.

Materials and methods: A cross sectional observational questionnaire-based study was conducted in a

tertiary care teaching hospital after taking ethical clearance from the Institutional Review Board. The study participants included all the prescribing physicians.

Results: $45 \%$ of the participants felt that the promotional literature always influenced their prescribing.

$70 \%$ preferred written media like pamphlets, brochures and leaflets as promotional literature when compared to electronic media. Most of the prescribing physicians found it difficult to find all the missing 


\section{FÎ́RMST}

4-5 Aug 2020 | Moscow

information from the drug promotional literature.

Conclusion: Prescribing physicians must be provided with training for critically analyzing such materials. Pharmaceutical companies should ensure that the published content is in accordance with the guidelines. Physicians must be aware of the risk of using non independent information sources.

Keywords: Drug promotion, drug marketing,ethics

Conflict of interests/Comments: None.

FIRMST20_AB_39

Non-Clinical Sciences - Pharmacology

\section{KNOWLEDGE, ATTITUDE AND PRACTICES OF RATIONAL USE OF MEDICINE AMONGST POSTGRADUATES AND INTERNS IN A TERTIARY CARE TEACHING HOSPITAL.}

Sakshi BHARATI1, Kavitha VIVEK1, Vaishal THAKARE1, Deepak LANGADE1

1DY Patil University School of Medicine, Navi Mumbai, India

Background: Prescribing is a complex and challenging task which must be based on accurate and objective information and not automated action. Rational use of drugs require that patients receive medication appropriate to their clinical needs, in doses that meet their own individual requirements for an adequate period of time and at lowest cost to them and their community. Worldwide; irrational Prescribing and polypharmacy have been identified as major cause of poor prescription. Hence, the present study was conducted with the aim to evaluate knowledge, attitude and practices of rational use of medicine amongst post graduates and interns in a tertiary care teaching hospital.

Methods: A cross sectional observational study was conducted among 100 postgraduate and interns at $\mathrm{Dr}$ D.Y. Patil School of Medicine, Navi Mumbai. Data was collected using a pre validated, self administered questionnaire. Descriptive statistics were used to analyse the data.

Result: A total of 100 interns and postgraduates participated in this study. 20\% always prescribed essential medicines, while $30 \%$ occasionally did so. $53 \%$ always prescribe medicine taking into consideration adverse effects, interactions and contradictions $24 \%$ prefer writing generic names of medicine while Prescribing, 34\% prefer trade names, $42 \%$ prefer both, $20 \%$ prefer prescribing old drugs, $10 \%$ prefer new drugs while $70 \%$ prefer both.

Conclusion: In the present study majority of respondents were not aware about most of the issues concerned with RUM addressing questionnaire which shows inadequate knowledge about the same, but being future prescribers all of them need to have adequate knowledge about all of the issues addressed. Although the concept of RUM is included in undergraduate curriculum more vigorous training regarding the same is required to strengthen the mechanism for continuing professional development of clinicians to update their knowledge and skills to prescribe drugs rationally.

Keywords: Rational use of medicines, Essential medicines, P-drug concept

Conflict of interests/Comments: None declared

FIRMST20_AB_38

Non-Clinical Sciences - Pharmacology

\section{QUALITY OF SLEEP PATTERN AMIDST THE COVID-19}

PANDEMIC: A CROSS-SECTIONAL OBSERVATIONAL STUDY

Shloka HEGDE1, NIMISHA, CHAUDHARY1, KAVITHA, DONGERKERY1, Vaishali THAKARE1

1DY Patil University School of Medicine, Navi Mumbai, India

Introduction/Background: It is a well known fact that sleep is a critical biological process for the proper functioning of the human body. Therefore when confronting the COVID-19 pandemic sleep has become even more essential. With such unprecedented changes coming so quickly, it is understandable that the importance of sleep is flying under the radar. Social isolation, increase in screen time, economic/work 


\section{FÎ́RMST}

4-5 Aug 2020 | Moscow

stress and fear of contracting the virus can all bring about a profound change in the normal routine of both frontline workers as well as general population, which can directly as well indirectly affect their sleep pattern more than usual.

Keeping this in mind the following study was conducted throughout India with the following aim.

Aim- To evaluate and analyse the change in sleep experienced by healthcare workers and general population during COVID-19 pandemic.

Materials and Methods- The following cross sectional questionnaire based observational study which was conducted in Dr D Y Patil University-Navi Mumbai.The study was conducted amongst healthcare workers and general population all over India through a prevalidated, pretested questionnaire which incorporated the Pittsburgh Sleep Quality Index and were sent to the candidates through a Google Docs Link.

Results- A total of 1030 candidates participated in this study. $74 \%$ agreed that an increase in screen time affected their sleep, however $56.9 \%$ believe that watching the news about the pandemic in this screen time did not. $61 \%$ of the candidates did not believe that the fear of contracting the virus affected their sleep however $59.1 \%$ believed that decrease in social interaction definitely did.Also from our study we learnt something interesting, about $6 \%$ of our candidates who did not consume medication to improve their sleep prior to the pandemic had begun to use it amidst the pandemic.

Conclusion- The following study has awakened us to the detrimental effect the COVID-19 pandemic has had on sleep. This study clearly shows that it's not the virus or the fear of the virus that has affected sleep, but basic requirements like decreased human interaction, showing ultimately that humans are simply social animals with needs like food, water, air and most importantly sleep.

Keywords: Pandemic,Sleep,Social Interaction,Sleep Medication

Conflict of interests/Comments: There is no recorded conflict of interest.

\author{
FIRMST20_AB_37 \\ Clinical Sciences - Surgery
}

\section{TELEPHONIC CLINICS: “A REVISIT DURING COVID-19”}

\section{Meghana TAGGARSI1, Subba Rao}

KANCHUSTAMBAM1,

1Royal Blackburn Hospitals, East Lancashire Teaching Hospitals, Blackburn, United Kingdom.

Background and Objectives: "How effective the TeleClinics are during COVID-19 pandemic? Any take home messages for the future?"

Methods: Pre-set questionnaires were distributed to surgical clinicians conducting tele-clinics; subsequently patients were interviewed for their feedback based on pre-set questions. Lastly nurses who otherwise help to run outpatient clinics were asked for their feedback.

Results: In this study, 15 clinicians and 12 patients were randomly interviewed. $46 \%$ of the clinicians and $83 \%$ of the patients agreed that telephonic consultation is simple, convenient, flexible and quick. Two thirds of clinicians felt that it requires less staffing and is cost effective. From the patient's perspective, more than $80 \%$ agreed that tele-clinics are more efficient - no more anxiety, especially related to waiting delays and parking. However, a minority (16\%) of patients expressed the stress built-up waiting for the call and lack of physical examination. As one would expect, most (86\%) surgeons opined that tele-clinics were a hindrance to detailed physical examination. Do these virtual clinics accelerate the actual process of overall management thus getting the definitive treatment early? Half the clinician responses were positive with a caveat that the patients could be under or over investigated. How did the surgeons perform from the patient's viewpoint? Most (91.6\%) patients were satisfied with the way their ailment was addressed. They felt the virtual clinics are quick, convenient; still some would prefer to meet their doctor to discuss the final management plan.

Interpretations and conclusions: Overall it was a satisfactory experience to surgeons as well as patients. Our study provides the evidence that it has been a satisfactory alternative to physical outpatient clinics during the times of "COVID-19", both to clinicians and patients. 


\section{FÎ́RMST}

4-5 Aug 2020 | Moscow

Keywords: Clinicians, COVID-19, nurses, patients , Tele-clinics, Virtual clinics.

Conflict of interests/Comments: None

FIRMST20_AB_36

Clinical Sciences - Surgery

\section{A NOVEL TECHNIQUE OF MINIMALLY INVASIVE RETRORECTUS REPAIR OF VENTRAL HERNIA- MODIFIED ETEP.}

Anil KUMAR1, Meghana TAGGARSI2, Subhash RC3 1Stepping Hill Hospital, Stockport NHS Foundation Trust, Stockport, United Kingdom

2Royal Blackburn Hospital, Blackburn, United Kingdom 3Devagiri Hospital, Bangalore, India

Introduction: Ventral hernia repair is one of the common general surgical procedures. A novel technique has been developed where a mesh is placed in a retro muscular plane by minimal access technique - Extended totally extraperitoneal repair (eTEP). There is scarcity of data on its effectiveness when compared to conventional laparoscopic intraperitoneal onlay mesh (IPOM) hernioplasty

Aims and Objectives: To compare the clinical effectiveness of eTEP technique versus conventional IPOM repair for ventral hernia. We also aim to introduce our innovative Kumar-Subhash's modified 3 port eTEP technique for repair of ventral hernias.

Materials and Methods: A prospective comparative study was undertaken at Devagiri Hospital, Bangalore, India from January 2017 to December 2019. 30 patients were included in the study based on predefined inclusion and exclusion criteria. Patients were divided into two groups, the eTEP group of 12 patients and 18 patients were included in the IPOM group. Since, we adopted a new technique, patients' choice of procedure was respected and hence, no randomisation was done. Appropriate statistical analysis of the collected data was done. The outcomes were compared with respect to postoperative pain, duration of hospital stay and postoperative complications.

Results: Both groups were comparable with respect to age, gender and presenting complaints. The mean pain score on postoperative day (POD) 1 in eTEP group was 3 , and 1 on POD 7 while the IPOM group had a mean pain score of 7 and 3 on POD 1 and POD 7 respectively which was statistically significant. Patients in the eTEP group had a shorter length of hospital stay. None of the patients in the eTEP group had any complications related to the novel technique.

Conclusion: Our study demonstrates that KumarSubhash's modified 3 port eTEP technique is a novel and easy approach for laparoscopic repair of ventral hernia especially umbilical hernia and offers an additional skill to the armamentarium of Minimally Invasive surgeons. It is a feasible, effective and safe option for repair of ventral hernias with good clinical and cosmetic outcomes for patients.

Keywords: eTEP, IPOM, ventral hernia, retrorectus, mesh.

\section{Conflict of interests: None}

FIRMST20_AB_35

Non-Clinical Sciences - Pharmacology

\section{ANALYTICAL QUESTIONNAIRE BASED STUDY TO}

EVALUATE THE PATTERN OF EATING DISORDER

AMONGST MEDICAL FRATERNITY VS GENERAL

POPULATION DURING COVID 19 PANDEMIC AND ITS

PILOT STUDY

Mahip DEVA1, Suhani AGRAWAL1, Vaishali

THAKARE1, Kavita DONGERKERY1, Anant PATIL1

1DY Patil University School of Medicine, Navi Mumbai, India

Introduction/background:

Eating disorders have been very common among the teenagers as well as adults in this time of covid-19 pandemic. Due to the lockdown there has been a lack of routine which leads to distorted eating habits and snacking throughout the day. Eating disorders among the medical fraternity is a very crucial matter as it will directly or indirectly affect the health of future 
doctors. Eating disorders accompanied with stress can also lead to anorexia nervosa, bulimia nervosa. Eating disorder is common against stressful situations within the affected people, mainly during COVID-19 pandemic and the people affected with it. Hence we had planned to conduct this observational, crosssectional study to compare the impact of COVID 19 pandemic on eating disorders amongst the doctors and general population

\section{Objective:}

To analyze the psychological impact of COVID-19 pandemic as an eating disorder among the medical fraternity.

Material and methods:

observational, cross sectional study was conducted in Dr. D.Y. Patil Hospital, Nerul, Navi Mumbai. Study population divided in two groups as medical \& general population. Medical group contains 16 participants while nonmedical includes 14 participants from the general population. Data was collected through a pre validated, pretested questionnaires which include Eat26 questionnaire, demographic details and few more

sections.

Results:

Total 30 participants included in this study divided them into two groups. In demographic $46 \%$ were female while $54 \%$ male. $25 \%$ participants in the medical group had $>20$ Eat 26 score, while in the nonmedical group it was higher in $7 \%$ participants. The mean score in the Medical group was 13.7 \& in the general population was 8.85 . This difference is statistically significant, $\mathrm{p}$ value 0.04 (unpaired $\mathrm{T}$ test).

Conclusion:

In the present study we have concluded that the risk of eating disorder as an impact of stress because of multiple factors of COVID -19 pandemic was higher in the medical group compared to the general population.

References:

https://www.verywellmind.com/emotional-eatingduring-covid-19-pandemic4802077 https://www.google.com/amp/s/www.psychologytod ay.com/us/blog/naked-truth/202003/how-curb- emotional-eating-during-the-covid-19-

pandemic\%3famp

https://www.google.com/amp/s/www.click2houston. com/health/2020/04/13/quarantine-15-binge-eatingcausing-weight-gain-during-

covid-19-pandemic/\%3foutputType=amp

Keywords: Medical, General population, Pilot Study, Eating disorder, Covid 19 Pandemic

Conflict of interests: There is no recorded conflict of interest

FIRMST20_AB_34

Clinical Sciences - Oncology \& Hematology

THE CLINICAL SIGNIFICANCE OF LABORATORY BIOMARKERS OF AN ANTITUMOR IMMUNE RESPONSE.

Anna MALKOVA1, Rashida ORLOVA1, Vladimir SHAROYKO1

1Saint Petersburg State University, Saint Petersburg, Russian Federation

Introduction: The antitumor immune response is provided by innate and adaptive immunity and aimed at the elimination of cancer cells. A breakthrough in cancer treatment was immunotherapy, based on activation of the patient's own immune system against tumor cells. Despite the pathogenic approach to the treatment and promising preclinical experiments, in practice, immune therapy was effective in up to $40 \%$ of cases (Azoury SC, 2015). The study aims to analyze possible immunological biomarkers of efficacy for treatment with checkpoint inhibitors.

Materials and Methods: Review and original articles published from 2005 to 2020 in the international databases PubMed, Web of Science, Elsevier were selected using the keywords: checkpoint inhibitors, immunotherapy, biomarkers, efficacy.

Results: The markers-predictors of high efficacy of therapy with checkpoint inhibitors were identified and classified according to pathogenic mechanism of drug action:

High mutagenic potential of the tumor itself, characterized by the level of tumor mutation load (TMB), evolutionary divergence of HLA-I (HED), a high level of mutations in the circulating tumor DNA; 


\section{FÎ́RMST}

4-5 Aug 2020 | Moscow

High activity of the adaptive immunity, which include low expression of inhibitory T-cell receptors, the presence of single nucleotide polymorphisms (SNPs) in genes encoding $\mathrm{F} c \gamma \mathrm{R}$ T-lymphocyte receptors, an increased number of tumor infiltrating lymphocytes (TILs), a high number of eosinophils and lymphocytes in peripheral blood (with predominance of CD8, CD4 memory $T$ cells and decreased T-regulatory cells), high concentration of II-2, VEGF-C and low concentration of II-10;

Decreased activity of the tumor microenvironment, characterized by a reduced number of myeloid suppressor cells (MDSCs), relative decreased neutrophils in biopsy material and peripheral blood, low levels of II- $1 \beta$, II-6, ANG2, C- reactive protein (CRP).

Conclusions: Determination of high rates (TMB), HED, high mutation rate in circulating tumor DNA before treatment can predict tumor response to the immune therapy, which can be used for more personalized patient selection. Biomarkers of innate and adaptive immunity may indicate the activity of tumor microenvironment and cytotoxic immunity. Determination of the markers before and during the treatment might be an appointment indication for various immunomodulatory drugs, inhibitors of proinflammatory cytokines and pro-angiogenic molecules.

Keywords: antitumor immune response, efficacy, biomarkers, adaptive immunity, tumor microenvironment, genetics

Conflict of interests: No Conflict of interests

FIRMST20_AB_33

Non-Clinical Sciences - Pharmacology

ANALYTICAL QUESTIONNAIRE BASED STUDY TO EVALUATE THE PATTERN OF EATING DISORDER AMONGST MEDICAL FRATERNITY VS GENERAL POPULATION DURING COVID 19 PANDEMIC AND ITS PILOT STUDY.

Mahip DEVA1, Suhani AGARWAI1

1Dr Dy Patil Navi Mumbai School of Medicine, Navi Mumbai, India

Introduction/Background: Eating disorders have been very common among the teenagers as well as adults in this time of covid-19 pandemic. Due to the lockdown there has been a lack of routine which leads to distorted eating habits and snacking throughout the day. Eating disorders among the medical fraternity is a very crucial matter as it will directly or indirectly affect the health of future doctors. Eating disorders accompanied with stress can also lead to anorexia nervosa, bulimia nervosa. Eating disorder is common against stressful situations within the affected people, mainly during COVID-19 pandemic and the people affected with it. Hence we had planned to conduct this observational, cross-sectional study to compare the impact of COVID 19 pandemic on eating disorders amongst the doctors and general population

Objective: To analyze the psychological impact of COVID-19 pandemic as an eating disorder among medical fraternity. Material and methods: observational, cross sectional study was conducted in Dr. D.Y. Patil Hospital, Nerul, Navi Mumbai. Study population divided in two groups as medical \& general population. Medical group contains 16 participants while nonmedical includes 14 participants from the general population. Data was collected through a prevalidated, pretested questionnaire which included Eat26 questionnaire, demographic details and few more sections.

Results: Total 30 participants included in this study divided them in two groups. In demographic $46 \%$ were female while $54 \%$ male. $25 \%$ participants in the medical group had $>20$ Eat 26 score, while in the nonmedical group it was higher in $7 \%$ participants. The mean score in the Medical group was $13.7 \&$ in the general population was 8.85 . This difference is statistically significant, $\mathrm{p}$ value 0.04 (unpaired T test).

Conclusion: In the present study we have concluded that the risk of eating disorder as an impact of stress because of multiple factors of COVID -19 pandemic was higher in the medical group compared to the general population.

\section{References:}

https://www.verywellmind.com/emotional-eatingduring-covid-19-pandemic4802077

https://www.google.com/amp/s/www.psychologytod ay.com/us/blog/naked-truth/202003/how-curbemotional-eating-during-the-covid-19-

pandemic\%3famp

https://www.google.com/amp/s/www.click2houston. com/health/2020/04/13/quarantine-15-binge-eating- 


\section{FÎ́RMST}

4-5 Aug 2020 | Moscow

causing-weight-gain-during-covid-19-

pandemic/\%3foutputType=amp

Keywords: Medical, General population, Pilot Study, Eating disorder, COVID-19 Pandemic.

Conflict of interests/Comments: There is no recorded conflict of Interest.

FIRMST20_AB_32

Non-Clinical Sciences - Pharmacology

EVALUATION OF EXPERIENCES, AWARENESS, ATTITUDE, AND PRACTICES AMONG UNDERGRADUATE STUDENTS OF HEALTH SCIENCES UNIVERSITIES TOWARDS E-LEARNING: NATIONAL QUESTIONNAIRE BASED OBSERVATIONAL STUDY

Tanusri TETARBE1, Hritika SHARMA1, Kavitha VIVEK1, Vaishali THAKARE1, Deepak LANGADE1

1Dr DY Patil School of Medicine, Navi Mumbai, India

Background: The COVID-19 Pandemic has created a lot of havoc in the history of mankind. Implementation of social distancing has forced many countries to go into complete lockdown and also led to a halt in the ongoing academic sessions of schools and colleges. ELearning has proved to be really helpful in terms of not halting the speed of education and maintaining the continuity. There are a few challenges that every student is facing with this sudden transition of methodology in learning.

Hence the present study was conducted with the aim to analyze the experiences and awareness among the undergraduate students towards E-Learning during COVID-19 pandemic and to evaluate the attitude and practices towards the online learning among them.

Methods: A National Cross sectional and Observational study was conducted among 1110 undergraduate students (1st to final years of different health sciences institutes in India) at Dr DY Patil, School Of Medicine, Navi Mumbai in the Pharmacology Department. Data was collected using a pre validated, self-administered questionnaire. Descriptive Statistics was used to analyze the data.

Results: A total of 1110 undergraduate students from 17 Different Health Sciences Institutes in India had participated in this study. $83.15 \%$ students suggested that a prior training of the faculty with the use of technology would improve the session. $64.77 \%$ students faced difficulty in understanding through online medium. $77.93 \%$ students prefer classroom teaching over Live Online Lectures and $61.62 \%$ would like to resume with classroom teaching post the pandemic.

Conclusions: In the present study we have concluded that even though $\mathrm{E}$ Learning has helped us and is the need of the hour, it cannot replace the traditional classroom learning as well as practical aspects of bedside teaching.

Keywords: COVID 19, E Learning, Medical Education.

Conflict of interests/Comments: None

FIRMST20_AB_31

Clinical Sciences - Internal Medicine

THE OCCULT RHEUMATIC SCOURGE: AN AUTOPSY ANALYSIS OF MISSED / SILENT RHEUMATIC HEART DISEASE.

Pawan DAGA1

1Seth GS Medical College, Mumbai, India

Aim and Background: Chronic rheumatic heart disease (RHD) is the major sequel of healing of acute rheumatic fever (ARF), which often results in crippling valvular deformities. The patients are usually young and present with features of mitral and/or aortic valvular dysfunctions. However, at times, the disease may remain asymptomatic and may be seen as incidental findings at autopsy or may even be a cause of sudden cardiac death. This paper aims to study the clinical and pathological manifestations of missed cases of RHD and postulate possible reasons behind a missed diagnosis.

Material and Methods: A retrospective 20-year (20002019) autopsy data of chronic RHD was reviewed and patients, in whom the valvular deformities had been incidental autopsy findings, were selected. The clinical details of these patients were correlated with morphology of the affected valves. On this pathological analysis, the patients were assigned to a 
category of stenosis with or without regurgitation and regurgitation. Also the cases were analyzed on the basis of subtle or significant valvular deformity and their clinical diagnosis.

Results: Among 475 cases of chronic RHD identified at autopsy in the study period, the disease was diagnosed incidentally in 69 patients ( $14.5 \%$ ). Significant valvular deformity was noted in 61 cases while others had subtle valvular deformity. The most common cause of death was cardiac failure (39 patients, 56\%). 11 cases had experienced sudden cardiac death. Amongst the undiagnosed cases, only 5 were clinically diagnosed with cardiac pathology while others had non cardiac overwhelming diseases which most commonly included acute febrile illness (most commonly pneumonia or fever of unclear etiology). The most common misdiagnosis was pulmonary thromboembolism and cardiomyopathy.

Conclusion: Our study indicates that mortality and morbidity due to RHD is under-determined. Patients remain undiagnosed due to either insignificant valvular involvement, clinically silent in the presence of significant valvular deformity, presence of other overwhelming diseases or misdiagnosis partly due to resemblance with other pathologies.

Keywords: RHD, Rheumatic Heart Disease, missed RHD.

Conflict of interests/Comments: None

\#FIRMST20_AB_30

Non-Clinical Sciences - Pharmacology
CHALLENGES, ATTITUDE, AND PRACTICES TOWARDS E-LEARNING SESSIONS FOR MEDICAL EDUCATION BY FACULTIES DURING COVID 19 PANDEMIC: A NATIONAL QUESTIONNAIRE BASED OBSERVATIONAL STUDY IN TERTIARY CARE TEACHING HOSPITALS

Tanusri TETARBE1, Hritika, SHARMA1, Vaishali

THAKARE1, Kavitha VIVEK1, Deepak LANGADE1

1Dr. DY Patil School of Medicine, Navi Mumbai, India

Background: The COVID-19 Pandemic has created a lot of havoc in the history of mankind. Implementation of social distancing has forced many countries to go into complete lockdown and also led to a halt in the ongoing academic sessions of schools and colleges. ELearning has proved to be really helpful in terms of not halting the speed of education and maintaining the continuity. There are a few challenges that every faculty is facing, with this sudden transition of methodology for teaching. Hence the present study was conducted with the aim of to evaluate the impact of sudden change in the teaching platform, attitude \& practices towards E-learning among medical faculties during COVID 19 Pandemic.

Methods: The present Cross-sectional, questionnaire based observational study was conducted in Dr. D.Y. Patil University, Navi Mumbai. The study was conducted amongst the medical teaching faculties all over India through pre validated, self-administered questionnaires which were sent through a Google docs link.

Results: A total of 215 faculties from 11 health science institutes had participated in this study. 64. 65\% participants experienced technical difficulties in conducting E- learning sessions, $74.88 \%$ had poor student teacher interactions, $26.97 \%$ had lack of motivation for preparation of classes and $47.90 \%$ participants conducted online examination for evaluation of theory \& practical skills.

Conclusions: In the present study we have concluded that even though E-learning was the need of an hour and will continue to be, it cannot replace traditional classroom and bedside teaching in the medical field.

Keywords: E-learning, COVID 19 Pandemic, Medical education, Medical faculties, Technical difficulties

Conflict of interests/Comments: No 


\section{FÎ́RMST}

4-5 Aug 2020 | Moscow

FIRMST20_AB_29

Non-Clinical Sciences - Public Health

\section{KNOWLEDGE, ATTITUDE AND PRACTICE OF SELF MEDICATION DURING COVID-19 PANDEMIC: A QUESTIONNAIRE BASED STUDY}

Hritika SHARMA1, Anant PATIL1, Tanusri TETARBE1

1Dr. D.Y. Patil University, School of Medicine, Navi Mumbai, India

Aim: To evaluate knowledge, attitude and practice of self-medication during COVID-19 pandemic

Material and methods: In this cross-sectional study, knowledge, awareness and practices about selfmedication during COVID-19 pandemic were recorded among the adult population. Prevalence of selfmedication during the last 3 months was calculated.

Results: A total of 630 participants (mean age 22.04 years; $69.21 \%$ females and $87.78 \%$ students) were included. A total of 106 (16.83\%) study participants consumed medication without a prescription from a qualified doctor in the last 3 months. Mean age of participants who consumed medications was significantly higher than those who did not consume self-medication [27.25 (+10.79) vs $21.18 \quad(+3.75)$; $\mathrm{p}<0.001]$. The difference between male and female participants who consumed self-medication was statistically significant $(p=0.0087)$. A total of 96 $(90.57 \%)$ consumed allopathic medication and 103 (97.16\%) used oral medicines. Common causes of selfmedication included pain 56 (53.8\%), menstrual problem 22 (20.8\%), muscle cramp 21 (19.8\%), fever $20(18.9 \%)$ and abdominal pain/burn/acidity 19 (17.9\%). A total of $102 \quad(96.22 \%)$ reported improvement in symptoms after self-medication and 5 (4.71\%) reported side effects. Two important reasons cited by study participants for self-medication included minor health problems $70(66 \%)$ and easy availability of medicines at the medical store 44 (41.5\%). According to 422 (66.98\%) study participants, penalty for self-medication should be based on the type of drug consumed.
Conclusion: A total of $16.83 \%$ study participants consumed self-medication during the previous three months. Increased awareness about self-medication is important to avoid complications associated with selfmedication.

Keywords: Awareness, COVID-19, General population, Self-medication.

Conflict of interests: None.

FIRMST20_AB_28

Non-Clinical Sciences - Public Health

KNOWLEDGE AND AWARENESS ABOUT COVID-19 AMONG GENERAL POPULATION

Suhani AGARWAI1, Anant D PATIL1, Dr. Vaishali

THAKARE1

1Dr. DY Patil School of Medicine, Navi Mumbai, India

Objective: To evaluate knowledge and awareness about Coronavirus Disease (COVID-19)

Material and methods: In this cross-sectional study, a pre-tested questionnaire consisting of questions related to knowledge and awareness about COVID-19 was administered to adult people in India.

Results: A total of 241 people (mean age 30.45 years; $56.85 \%$ male) participated of whom 214 (88.80\%) were aware that COVID-19 is a primarily respiratory illness and 237 (98.34\%) were aware about spread of coronavirus from person to person. A total of 235 $(97.51 \%)$ were aware that recovery is possible from COVID-19 and 225 (93.36\%) were aware that there is no vaccine for protection against it. A total of 221 (91.70\%) participants mentioned that coronavirus can spread by coughing, sneezing as well as touching the contaminated objects. According to 221 (91.70\%) participants, a person with a positive test for coronavirus is a confirmed case and 189 (78.42\%) reported that a patient having a positive test for 
coronavirus, but no symptoms are an asymptomatic carrier. According to 223 (92.53\%) elderly people are at higher risk of developing more complications due to COVID-19. According to 239 (99.17\%) participants washing hand with soap and water for $>20$ seconds and avoiding close contact with sick people is important for prevention of spread of virus. According to $210(87.14 \%)$, masks help in protection of self and others. According to 180 (74.69\%) participants lockdown is useful for breaking the chain of transmission of virus.

Conclusion: Knowledge and awareness about most of the aspects of COVID-19 disease is good among the study participants.

Keywords: Awareness, COVID-19, knowledge.

Conflict of interests: None.

FIRMST20_AB_27

Clinical Sciences - Ophthalmology

\section{VISUAL ACUITY CHANGES ASSOCIATED WITH SHUNT MALFUNCTION AND OPTIC ATROPHY.}

Antonina POPOVA1, Maria PAVLIV1

1I.M. Sechenov First Moscow State Medical University, Moscow, Russian Federation

Introduction: One of the main signals of dysfunctions in the brain are changes in the embryologically connected organ-the eye. Consequently, congenital pathologies of the brain, including hydrocephalus, will directly affect the functions of the eye. The most serious complication of hydrocephalus can be optic atrophy.But is it possible to prevent the appearance of optic nerve atrophy (ONA) in complex clinical cases? If the shunt broke, but there are no obvious signs of increased intracranial pressure (ICP), and changes in the fundus of the eye?
Materials and methods: We studied 6 cases of hydrocephalus with different kinds of blindness to determine the correlation between the time of diagnosis of the shunt dysfunction with the visual acuity in the period from several hours to more than a year.

Results: Our analysis of individual case histories of patients with shunt malfunction indicated: various types of blindness plays several parts. Firstly, transient visual loss as a diagnostic character of a shunt dysfunction,can last for a few hours, associates with memory loss and nausea [9].Secondly, reversible visual loss as a result of shunt malfunction. An improvement of vision can occur over a year since shunt replacement, be only monocular. This type of blinded associates with normal pupillary reaction and fundoscopy, sense of retro-orbital pressure, weakness $[10,11]$.Thirdly,permanent blindness as a result of untimely treatment. This kind of outcome is shown in the case which we personally described.After shunt system malfunction the new one has been placed after half a year. In this period of time total ONA on the right side occurred, partial ONA-on the left.There were neither visual, nor brain disorders.

Conclusion: Blindness occurs as the diagnostic character of an ineffective treatment of hydrocephalus or due to it.Based on our analysis, it is possible to establish a certain symptoms pattern before vision loss, but it can be unpredictable.We suggest that reason for temporary total blindness is time of high ICP correction.Additional studies are necessary in order to determine the symptoms of future blindness and factors of possible vision return.It will help to improve the treatment algorithm and to decrease patient's disablement.

Keywords: Optic atrophy; visual loss; hydrocephalus; complication of hydrocephalus; shunt

Conflict of interests: The authors declare no conflict of interest.

FIRMST20_AB_26

Clinical Sciences - Rheumatology 


\section{FÎ́RMST}

4-5 Aug 2020 | Moscow

\section{TAKAYASU ARTERITIS AND SACROILIITIS: A CASE- CONTROL STUDY. \\ Francesca REGOLA1, Franco FRANCESCHINI1, Angela TINCANI1, Paola TONIATI1}

1ASST Spedali Civili and University of Brescia, Brescia, Italy

Introduction: A possible shared immunopathogenesis between Spondyloarthritis (SpA) and Takayasu Arteritis (TA) has been hypothesized and some clinical cases about SpA in TA patients have been reported (1). In clinical practice, the diagnosis of sacroiliitis may be performed by X-ray, Computed Tomography (CT) or Magnetic Resonance Imaging (MRI). In particular, CT findings of sacroiliitis include contour irregularities, joint space alterations, joint erosion, subchondral bone changes (osteoporosis or sclerosis), enthesitis, ankylosis. Meanwhile, TA patients had routine FDG$\mathrm{PET} / \mathrm{CT}$ scans for monitoring disease activity.

This study aims to see if there is an increased incidence of sacroiliitis in TA patients.

Material and methods: We collected retrospectively, imaging data from FDG-PET/CT scans of 28 TA patients and 28 controls, matched for sex and age. Controls were selected among patients who had FDG-PET/CT in our Nuclear Medicine Unit, excluding patients with bone tumors, bone metastasis and thyroid cancers. The majority of controls were affected by lymphoma in complete remission. An expert rheumatologist read the CT-scans of sacroiliac joints.

Results: No patients or controls demonstrated FDGuptake in sacroiliac joints.

In the control group we detected sacroiliac sclerosis in two cases: one due to degenerative changes, one to sacroiliitis $(1 / 28,4 \%)$.In the TA group four patients presented CT alterations suggestive for sacroiliitis: one bilateral erosion, one bilateral sclerosis, two monolateral sclerosis $(4 / 28,14 \%)$. One of these patients complained an inflammatory back pain.

Conclusion: In our cohort of TA patients we demonstrated an increased prevalence of sacroiliitis, diagnosed by CT scan. Only one patient reported an inflammatory back pain, while three patients had radiological signs of previous sacroiliitis. These findings highlight the importance of looking for spondyloarthropathy in TA patients even if asymptomatic.

(1): Guzel Esen S, Joint Bone Spine, 2019

Keywords: Rheumatology, Takayasu, Spondyloarthritis.

Conflict of interests: The authors declare no conflict of interest.

\#FIRMST20_AB_25

Clinical Sciences - Rheumatology

\section{C4 LEVELS AS A PREDICTOR OF FLARES AND ADVERSE PREGNANCY OUTCOMES IN PREGNANT WOMEN WITH SYSTEMIC LUPUS ERYTHEMATOSIS}

\section{Francesca CRISAFULLI1, Laura ANDREOLI1, Sonia} ZATTI1, Franco FRANCESCHINI1, Angela TINCANI1

1ASST Spedali Civili and University of Brescia, Brescia, Italy

Introduction: Pregnant women with SLE have an increased risk of Adverse Pregnancy Outcomes (APO). In clinical practice, low C3 and C4 levels are associated with active disease and during pregnancy, complement activation products are shown to be associated with APO. Our aim was to analyze C3 and C4 variations during SLE pregnancies, focusing on APO and disease flares.

Methods: Data on pregnant women with SLE, who were prospectively followed by a multidisciplinary team from 1987 to 2017, were analyzed. Serum C3 and C4 levels were recorded at preconception visit (TO) and each trimester (T1, T2, T3). Hypocomplementemia was defined according to the normality range calculated in healthy pregnancies1. APO were defined as: early miscarriage, intrauterine fetal death (IFD), perinatal death, pre-eclampsia (PE), severe preterm birth. Continuous variables are reported as mean

Results: 153 pregnancies in 106 women with SLE were analyzed. APO occurred in 25 (16\%): 13 early miscarriages, 4 IFD, 3 severe preterm births, 7 PE (hesitated in 2 IFD and 7 live births); 13 flares ( 2 renal, 


\section{FÎ́RMST}

4-5 Aug 2020 | Moscow

4 articular, 6 cutaneous and 1 neurological) were recorded in $11(7 \%)$ pregnancies. In pregnancies with flares C3 was lower at T2 and T3 as compared with pregnancies without flare (83.8vs105.3, $p=0.01$; 97.5vs115.5, $p=0.01$ ) while C4 was lower at T0 and T3 (8.6vs13.7, $p=0.02 ; 11.4 v s 16.9, p=0.006$ ). Considering the overall pregnancies, C3 progressively increased from T0 to T3, while it remained stable between T2-T3 in the flare group and between T0-T1 and T2-T3 in the APO group. In the flare group, C4 increased only between TO-T1 while in the APO group it remained stable throughout pregnancy. Notably, in pregnancies without APO, C4 increased from T0 to T2.

In pregnancies with APO, the variation of C4 levels between T2-T3 trimester was lower than in pregnancies without APO $(-2.98 v s 0,4 ; p=0.03)$. The frequency of low C4 was higher in pregnancies with flare at T0 and at each trimester as compared with pregnancies without flares ( $86 \%$ vs $21 \%, p<0.001$ ).

Conclusions: Low C4 at pre-conceptional visit seemed to predict flares during pregnancy. Lower increase of C4 levels between the 2 nd and the $3 r d$ trimester could predict APO.

References: 1Reggia R. et al. Rheumatology 2012; 51:2186-2190.

Keyword: Systemic Lupus Erythematosus; Pregnancy; Complement

Conflict of interests/Comments: No

FIRMST20_AB_23

Non-Clinical Sciences - Public Health

\section{E-CIGARETTES ALL SMOKE AND NO FIRE?}

Dominic Adam WORKU1, Elliott WORKU2

1St Georges Hospital, London, United Kingdom

2Prince Charles Hospital, Brisbane, Australia.

Introduction: E-cigarettes are an alternative to traditional tobacco-based cigarettes. While having considerable societal awareness, conflicting evidence exists to support their claims that they are an effective smoking cessation tool and are safe. Currently $>7000$ flavours exist with evidence that they exhibit detrimental cellular and tissue effects. A literature review was conducted utilising PubMed and Google Scholar Databases identifying papers between 2014 and 2019.

Aims of this study were to accurately gauge the safety and efficacy of e-cigarettes as a smoking cessation tool.

Methods: Search terms including 'electronic cigarettes' and 'vaping' were used to identify suitable references. A total of 314 articles were identified from which papers were excluded due to risk of bias, insufficient detail or were duplicate from which 58 papers were used in the final review.

Results: Evidence shows that e-cigarettes can have detrimental effects on several cell lines and animal models with their flavourings and nicotine content implicated; this has, however, not translated into major health outcomes after 3.5years follow-up but has been linked to chronic lung disease and cardiovascular disease. While advertised as an effective smoking cessation tool, no consensus can be made regarding their effectiveness although the first robust randomised controlled trial reports some success. This, however, is offset by the fact that the most common e-cigarette use is as a dual user and that there is evidence of threefold increased risk of future tobacco smoking.

Conclusion: Future research is needed to evaluate the long-term health outcomes and efficacy of e-cigarettes as a smoking cessation tool with greater discussion between patients and clinicians regarding this smoking cessation tool.

Keywords: E-cigarettes, Smoking cessation, nicotine, electronic nicotine delivery system

Conflict of interests: This work was published in SAGE open medicine journal.

FIRMST20_AB_22 


\section{FÎ́RMST}

4-5 Aug 2020 | Moscow

Clinical Sciences - Surgery

\section{PLEIOTROPIC EFFECTS OF STATINS - A MINI LITERATURE REVIEW.}

\section{Uzma SADIA1}

1University of North Midlands, Stoke on Trent. United Kingdom.

The use of statins has long been a topic of much controversy and debate. Because of the potential pleiotropic effects, statin therapy is currently being considered for medical conditions beyond cardiovascular disease. The lack of a definitive biomarker for statin pleiotropy makes it a challenge. This mini literature review aims at visiting the recent clinical trials with an emphasis on evidence for pleiotropy. As a practising vascular and endovascular surgeon, I find this topic fascinating and would like to open it for further discussion on this platform.

Keywords: Statins, pleiotropy, cardiovascular disease. Conflict of interests: None

FIRMST20_AB_21

Clinical Sciences - Oncology \& Hematology.

SUCCESSFUL EXPERIENCE OF TREATMENT OF THE PRIMARY MULTIPLE MALIGNANT TUMOURS (PMMT): RETINOBLASTOMA, OSTEOSARCOMA. CLINICAL CASE.
Albina ZAGIDULLINA1, Vakhtang KHARBEDIA2, Dmitriy NISICHENKO2, Svetlana, MIKHAILOVA2, Tatiana USHAKOVA2, Aslan DZAMPAEV2

1Pirogov Russian National Research Medical University (RNRMU), Moscow, Russian Federation

2Federal State Budgetary Institution «N.N. Blokhin National Medical Research Center of Oncology» of the Ministry of Health of the Russian Federation (N.N. Blokhin NMRCO), Moscow, Russian Federation

Retinoblastoma (hereinafter the "RB») is a malignant neoplasm, which has a very high risk of secondary tumour formation. The primary multiple malignant tumors (hereinafter the «PMMT») are the development of two or more histologically distinct neoplasms in the same patient, the existence of which poses a problem for oncologists.

Aim: to estimate the role of risk-factors in the treatment of PMMT; to evaluate the results of treatment.

Materials and methods: In our article, we will consider the clinical case of a patient with retinoblastoma, who developed a secondary neoplasm, an osteoblastic variant of osteosarcoma during treatment.. The RB1 gene was found both in the boy and his father. At the age of 2 years, he was diagnosed with bilateral retinoblastoma, OD stage T3bm, OS T3CNOMO, got special treatment in the period of $09.2005-11.2006$. In 2012 the child was operated twice: for cataracts and dissection of the posterior lens capsule. After 6 years, in 08.2018 , patient was diagnosed with osteosarcoma. He received general treatment of osteosarcoma with Doxorubicinum, Cisplatinu and, Methotrexate. On 07.03.2019 patient underwent surgical resection of the distal part of the right radial bone with replacement of the defect with an endoprosthesis of the wrist joint. Prosthesis used for this patient was individual, without mechanisms of distraction. A histological examination of postoperative material revealed the osteosarcoma of the distal metaphysis of the radial bone with signs of therapeutic pathomorphosis of the 4 th degree.

Results: The early postoperative period was uneventful. Now the patient feels himself satisfactory, does not have any complaints and is in remission for the 1 year. 
Conclusion: The etiology of the second tumors includes such factors as genetic determination, type of treatment for the first malignant neoplasm, the influence of environmental factors, and a combination of any of these factors. That's why it is extremely important to assess the risk of developing PMMT in the moment of primary RB manifestation and, depending on this, choose the right strategy of the therapy.

Keywords: retinoblastoma, osteosarcoma, neoplasms, endoprosthesis, radiotherapy.

Conflict of interests: No conflicts of interests

FIRMST20_AB_20

Clinical Sciences - Oncology \& Hematology

\section{CLINICAL SIGNIFICANCE OF THE MOST COMMON MUTATIONS IN THYROID CANCER.}

Polina FEDOROVA1, Vladimir NAZAROV1

1l P Pavlov First State Medical University of St. Petersburg, Saint Petersburg, Russian Federation

Introduction: Thyroid cancer (TC) is the most prevalent cancer among malignant neoplasms of the organs of the endocrine system. Cytological categories Bethesda III-V of TC is considered to be the most difficult to diagnose. Molecular genetic tests can be a tool to complement routine cytopathological studies.

The aim of the study: The aim of the study is to develop a panel for detecting point mutations in the cytological material of thyroid tumors using the multi-target single nucleotide elongation (MSE) method.

Materials and methods. The studied group of patients included 52 patients with thyroid neoplasm. Patients were divided into subgroups in accordance with Bethesda category IV, V and VI. The first subgroup consisted of 24 people, the second- 7, the third- 21 people. Cytological material was obtained by fineneedle aspiration biopsy. Genetic testing was carried out using MSE approach. Three panels were created, that included the most common mutations in thyroid cancer: mutations in the BRAF, KRAS, NRAS, HRAS genes. The results of a postoperative histological examination were obtained: 11 samples of papillary thyroid cancer, 9 samples of the follicular variant of papillary cancer and 5 - follicular adenoma.
Results: Mutations were detected in 39\% of cytological samples. BRAFV600E mutation was detected in $29 \%$ of found aberration (specificity- 100\%), NRASQ61R mutation in $10 \%$ of those. To verify the method, positive and negative control samples confirmed by PCR were used. In all control samples, the results were completely consistent with the molecular genetic study by the MSE method.

Conclusion: MSE is a highly sensitive and promising method for detection of mutations in cytological samples in patients with TC.multi-target single-base elongation (MSE), thyroid cancer, somatic mutation.

Keywords: multi-target single-base elongation (MSE), thyroid cancer, somatic mutation.

Conflict of interests: Difficulties of diagnostic thyroid cancer can be resolved by molecular genetic testing.

FIRMST20_AB_19

Non-Clinical Sciences - Pharmacology

\section{A COST VARIATION ANALYSIS OF VARIOUS BRANDS}

OF ORAL ANTI-DIABETIC DRUGS CURRENTLY

\section{AVAILABLE IN INDIAN PHARMACEUTICAL MARKET.}

\section{Sharvari SHYAM1, Bhavya DARSHINI.M2}

1BGS Global Institute of Medical Sciences, Bengaluru, India

2Bangalore Medical College, Research Institute, Bengaluru, India

Background: Diabetes Mellitus is a chronic metabolic disorder requiring lifelong treatment. There are a large number of anti-diabetic drugs available in the Indian market. The cost of drugs plays an important role in patient's care, warranting the need for all physicians to keep themselves updated with the latest prices and price variation of various brands of anti-diabetic drugs.

Objective: To evaluate the cost of oral anti-diabetic drugs of different brands currently available in the Indian market. 
Materials and Methods: Cost of oral anti-diabetic drugs manufactured by different pharmaceutical companies in the same strength and dosage forms was obtained from "Current Index of Medical Specialties" (CIMS) April- July 2019 and Indian Drug Review Vol. XXV - Issue 3 2019. The percentage price variation and cost ratio were calculated.

Results: The cost of 12 drugs in single therapy and 10 drugs in combination therapy were evaluated for percentage price variation. For single and combination therapy, a total of 68 different formulations manufactured by various companies were available. In sulfonylurea group of drugs, maximum price variation of $1366 \%$ was seen in Glimepiride $1 \mathrm{mg}$ and minimum price variation of $17 \%$ was seen in Glipizide $2.5 \mathrm{mg}$. In non-sulfonylurea group of drugs, a maximum variation of $809 \%$ was seen in Metformin $500 \mathrm{mg}$, followed by Voglibose $0.3 \mathrm{mg} 793 \%$ and a minimum variation of $10 \%$ in Acarbose 100mg. Among the fixed dose combination therapy, the highest price variation of $555 \%$ was seen in Glimepiride $2 \mathrm{mg}+$ Metformin $500 \mathrm{mg}$ shows and the least price variation of $8 \%$ was seen in Pioglitazone $7.5 \mathrm{mg}+$ Metformin 500mg combinations.

Conclusion: Our study showed that there is a high price variation for oral anti-diabetic drugs by different brands available in the Indian market. Since diabetes mellitus is a chronic illness, cost of the drug plays an important role in compliance to the treatment regimen. It is necessary to bring awareness among the physicians, pharmaceutical companies and the regulatory bodies regarding this wide variation in prices, such that the drug costs can be reduced and made more affordable to the common man.

Keywords: Antidiabetic drugs; Brands; Cost analysis; Cost variation; Price variation.

Conflict of interests: None

FIRMST20_AB_18

Non-Clinical Sciences - Microbiology
DEUS EX MACHINA: SYSTEMATIZATION OF CURRENT INFORMATION ON HELICOBACTER PYLORI INFECTION.

\section{Herman KISELEV1, Cyrill GORLENKO1}

1Sechenov University, Moscow, Russian Federation

Since discovery, Helicobacter infection is known to be a risk factor for gastritis, peptic ulceration, GIT tumors and numerous extragastric diseases. Throughout the time knowledge about pathogenesis of Helicobacter infection have been expanded with detection of multiple virulence factors related to invasion, adhesion and cytotoxicity such as VacA, CagA, BabA, OipA and last but not least, urease. Invasive and NonInvasive methods of diagnostics (e.g. Rapid Urease Test, Endoscopy, RT-PCR, different Serological Methods, Stool Antigen Test and Urea Breath Test) currently are being improved in effectiveness and accuracy. But still, due to different factors (e.g. dramatically increasing drug resistance), eradication of H.pylori with classic therapy with combination of Metronidazole, Amoxicillin and Bismuthate Tripotassium Dicitrate remains world-wide problem. We dedicate our literature study to systematization of modern data on pathogenesis, diagnostics and treatment of Helicobacter infection.

Keywords: Helicobacter pylori, Urease, Urea Breath Test, Rapid Urease Test

Conflicts of interests: Authors declare no conflict of interest.

FIRMST20_AB_17

Clinical Sciences - Surgery 


\section{FÎ́RMST}

4-5 Aug 2020 | Moscow

TREATMENT OF ADVANCED LARYNGEAL CANCER.

\section{Rogerio DEDIVITISI}

1University of Sao Paulo School of Medicine, Sao Paulo, Brazil

Since the beginning of the 1990s, non-surgical radiochemotherapy treatment has become popular with the prospect of maintaining oncological results and preserving the organ in patients with advanced squamous cell carcinoma of the larynx and hypopharynx. However, subsequent studies demonstrated increased recurrence and mortality after the non-surgical treatment became popular. More advanced tumors (T4) present better oncological outcomes when undergoing surgical treatment. For T3 tumors, organ preservation protocols present similar oncological results, with possibility of laryngeal preservation. However, preservative operations are an option with good oncological and functional outcome.

Keywords: Laryngeal cancer; laryngectomy; organ preservation protocol

Conflicts of interests: Nothing to declare.

FIRMST20_AB_16

Non-Clinical Sciences - Nuclear Medicine

THE ROLE OF THE KI-67 MARKER IN ASSESSING THE QUANTITATIVE DEGREE OF MALIGNANCY OF BREAST CANCER IN PET / CT STUDY.

TATSIANA, KAVALENKA, N.N. ALEXANDROV

NATIONAL CANCER CENTRE OF BELARUS.

Tatsiana KAVALENKA1

1N.N. Alexandrov National Cancer Centre of Belarus, Liasny, Belarus

Purpose: To evaluate the effect of the Ki-67 marker in breast cancer on the SUV max (a standardized uptake value), its quantitative assessment of the metabolic activity of the tumor process by PET / CT examination.

Methods and materials: Retrospective analysis of PET / CT studies from 2018 to 2019 with initial detection of breast cancer and lack of treatment.

Results: 80 patients were analyzed. All patients underwent the study with tracer 18F-FDG, the blood glucose level was 3.8-7.4 mmol/l, Ki-67 was from $3 \%$ to $90 \%$. Patients were divided into groups according to the Ki-67 level and the degree of malignancy of the tumor process $(G)$,.

The first group reflects the percentage of patients with a certain degree of malignancy of the tumor process on the Grade scale $(G)$ relative to the total number of patients and maximum value of SUV max:

$$
\begin{aligned}
& \text { G1 }<20-7.5 \% \text {, SUV max 3.6; } \\
& \mathrm{G} 1 \geq 20-0 \% \text {; } \\
& \mathrm{G} 2<20-21.25 \% \text {, SUV max 16.6; } \\
& \text { G2 } \geq 20-50 \% \text {, SUV max 20.31; } \\
& \mathrm{G} 3<20-0 \% \text {; } \\
& \text { G3 } \geq 20-10 \% \text {, SUV max 13.4; } \\
& \mathrm{G} 2-\mathrm{G} 3<20-0 \% \text {; } \\
& \text { G2-G3 } \geq 20-11.25 \% \text {, SUV max } 32.5 \text {. }
\end{aligned}
$$

The second one shows the minimum value of SUV max:

$$
\text { G1 }<20 \text { SUV max was 1.29; }
$$

$\mathrm{G} 1 \geq 20-0 \%$;

$\mathrm{G} 2<20$ the minimum value of the SUV max was 1.39;

$\mathrm{G} 2 \geq 20$ the minimum value of the SUV max was 1.34;

$\mathrm{G} 3+20-0 \%$;

$\mathrm{G} 3 \geq 20$ the minimum value of the SUV max was 1.9;

G2 - G3 - 20-0\%;

$G 2-G 3 \geq 20$, the minimum value of the SUV max was 1.85 .

Within these groups, clear dependence on the predicted increase in SUV max accumulation with the highest numerical value of Ki-67 was not observed. In one case, SUV max was 32.5 with $\mathrm{Ki}-67$ - $70 \%$ (G2-3); in another one, it was 5.48 (G2) for the same numerical values of Ki-67 ; whereas SUV max 16.6 with Ki-67 $10 \%(\mathrm{G} 2)$.

Conclusion: The high level of Ki-67 does not always determine its high metabolic activity and does not always characterize the degree of aggressiveness of the tumor process. It gives only a more likely result of increasing standardized uptake value.

Keywords: Breast cancer; Oncology; Ki-67 marker; PET / CT; Nuclear Medicine.

Conflicts of interests: Nothing to disclose. 
FIRMST20_AB_15

CLINICAL SCIENCES - IMMUNOLOGY

\section{ROLE OF ANGIOTENSIN RECEPTORS BLOCKERS in treatment of COVID-19.}

\section{Debraj GHOSH1, Tukakula Swetha REDDY1}

1People's Friendship University of Russia, Moscow, Russian Federation

Role of Angiotensin receptors blockers(ARBs) towards preventing COVID-19 infection in human cells by Debraj Ghosh and T. Swetha Reddy, People's Friendship University of Russia (RUDN), Moscow.

The global pandemic of COVID-19 is getting worse day by day and studying about the virus, we came up with certain ideas that can help in controlling and curing patients from this viral infection.

According to the structural biology of SARS-CoV-II virion, it has 4 structural proteins known as the $S$ (spike) glycoprotein, E (envelope), M (membrane) and $\mathrm{N}$ (nucleocapsid) protein. The spike protein is the one which is responsible to bind with the receptors in the host cells. It binds with Angiotensin converting enzyme 2 (ACE2) on human cells and invades the human cells through it. Also, Transmembrane protease, serine 2 (TMPPRSS2) is essential for entry of SARS-CoV-2. After fusion, an endosome forms around the virion and cathepsin cleaves it which in result in releasing the viral genome to enter the cell and start producing copies of the virus and thus infecting more cells. Thus if we use Angiotensin converting Enzyme 2 (ACE2) inhibitor drugs or Angiotensin receptor blockers (ARBs) (which we use to treat cardiovascular diseases such as losartan, valsartan, telmisartan, etc.), then we can prevents invasion of the virus into the cells.

Thus Angiotensin receptor blockers have effects that are similar to ACE inhibitors and hence it prevents attachment of SARS-CoV-2 RBD to ACE-2 expressing cells, thus inhibiting the viral infection to host cells.

Keywords: Spike glycoprotein (S), Angiotensin Converting Enzyme (ACE), Angiotensin Receptors blockers (ARBs), Transmembrane Protease, Serine 2 (TMPPRSS2), SARS-CoV-2.

Conflicts of interests: Thus, Angiotensin receptor blockers have effects that are similar to ACE inhibitors and hence it prevents attachment of SARS-CoV-2 RBD to ACE-2 expressing cells, thus inhibiting the viral infection to host cells.

FIRMST20_AB_14

Clinical Sciences - Obstetrics \& Gynecology

\section{RESISTANCE TO ANTIBIOTICS OF STAPHYLOCOCCUS SPECIES ISOLATED FROM WOMEN WITH RECURRENT MISCARRIAGE OF PREGNANCY.}

Maria LEVCHINA1, Viktoria Voevodina1, Elina

GREGORIANZ1, Elina KRAVCHUK1

1Tver State Medical University, Tver, Russian Federation

Introduction: One of the most important problems nowadays is recurrent miscarriage. Such pathology in Russia could be in the range of $10.5 \%$ of all pregnancies according to WHO. Different researches have shown that staphylococcus strains take in direct development of inflammatory processes in reproductive organs. Treatment of inflammatory processes is by antibacterial drugs. Specific mechanisms of resistance are developed due to uncontrolled use of antibiotics 


\section{FÎ́RMST}

4-5 Aug 2020 | Moscow

that can lead to decrease in effectiveness of treatment.

Aim: To assess antibiotic resistance in Staphylococcus aureus \& Staphylococcus epidermidis using serial dilution method among women with recurrent miscarriage in pregnancy.

Materials and Methods: Smears from the vagina of 33 women (age 35-40) with recurrent miscarriage in pregnancy were collected in transport medium and delivered into bacteriological laboratory within 2 hours. API systems (BioMerieux, France) were used for identification of Staphylococcus species. Serial dilution method was used to evaluate staphylococcus resistance against 10 antibiotics: oxacillin, vancomycin, erythromycin, tetracycline, clindamycin, ciprofloxacin, chloramphenicol, gentamicin, fusidic acids, and mupirocin with the help of EUCAST standards.

Results: 30 species of staphylococcus were identified: S.aureus (10)

S.xylosus (10)

S.epidermidis (10)

Most strains of S.aureus showed resistance to chloramphenicol (in 100\% of cases), gentamicin (90\%), tetracycline $(70 \%)$, oxacillin $(70 \%)$, erythromycin (60\%), fusidic acids (60\%) and ciprofloxacin (60\%). Strains of S.epidermidis were resistant to chloramphenicol (100\%), gentamicin (100\%) and ciprofloxacin (60\%). Strains of S.xylosus showed resistance to erythromycin (100\%), gentamicin (100\%), chloramphenicol (80\%), tetracycline $(70 \%)$, oxacillin (70\%), clindamycin (70\%), mupirocin (70\%).

Conclusions: From our work, we observed an increased tendency to antibiotic resistance among vaginal staphylococcus strains isolated from women with recurrent miscarriage in pregnancy. Almost all strains of $S$. epidermidis, S.aureus and S.xylosus showed resistance to gentamicin and chloramphenicol. Absolutely all strains of S.xylosus were resistant to erythromycin. We investigated a decreased tendency to staphylococcal sensitivity among the rest antibiotics. The results for such high resistance is probably due to the adaptation of microorganisms caused by their genetic variability, including accumulation of mutations and protective mechanisms of protein synthesis by cells of S.aureus and S.epidermidis.

Keywords: antibiotic resistance, recurrent miscarriage in pregnancy, serial dilution method, staphylococcus strains, antibacterial drugs.

Conflicts of interests: There is no conflict of interests. FIRMST20_AB_13

Non-Clinical Sciences - Neurosciences

\section{BRAIN STIMULATION - AN AVENUE FOR THERAPY OF MEMORY DISORDERS AND IMPROVISING MEMORY}

\section{Ashwin SAIKUMAR1}

1Pirogov Russian National Research Medical University, Moscow, Russian Federation

Introduction: The introduction of electrodes into various memory centres of the brain or by magnetic stimulation, it aims to improve the memory of patients with varying memory disorders and help humans achieve a heightened memory.

Materials \& Methods: Using data and experiments performed on patients while isolating those done exclusively for parts of the brain involved with episodic, semantic or autobiographical memory (AM). Also looking into patients who have a defect that gives them superior AM ( hyperthymesia) and those on the other end of the spectrum. Looking at the effects of memory caused due to stimulation both electrode and magnetic on the different parts of the brain relating to memory. Discerning ways of optimization of stimulation via electrodes.

Results:

MAGNETIC:

Healthy, younger and older participants on receiving a repetitive trans-cranial magnetic stimulation ( $r$-TMS), performed better on a memory task than during an $\mathrm{r}$-TMS-like placebo.The brain stimulation reaped similar benefits for the younger and older patients.

ELECTRIC: This kind of stimulation is done by insertion of hair-thin electrodes into the brain to deliver electricity and surge the activity of the basal nucleus of Meynert.Using intermittent stimulation - currently not used in any application for brain in patients - the monkeys were able to remember things up to five times longer in a standard test of working memory. 
The optimal results were obtained at 60 pulses per second for 20 seconds followed by a 40 - second interval without stimulation.An impairment of memory, of adult non-human primates, occurred on provision of continuous deep brain stimulation like the type used for Parkinson and currently under study on humans with Alzheimer's.

14 epilepsy patients who were scheduled to undergo surgery and required inserting intracranial depth electrodes to monitor seizure activities during the procedure, consistently remembered objects better when those objects had been paired with amygdala stimulation.

There is a problem with this method as it is invasive, requiring brain surgery to implant electrodes, but it is difficult to ensure that the electrodes are placed where you need them to be, to elicit a response. Thus, optimization is key.

Conclusion: These results prove that if stimulation is optimized, there is a possibility to improve a patient's memory by localizing the area in the brain that has degraded and provision of stimulation (magnetic or electric) has the possibility to improve degenerative memory pathologies.

1. https://www.dana.org/article/beyond-emotionunderstanding-the-amygdalas-role-in-memory/ 2.

https://www.sciencedaily.com/releases/2017/04/170 420141854.htm

3. http://www.scholarpedia.org/article/Episodic_memo ry

4. https://pubmed.ncbi.nlm.nih.gov/26726963/

Keywords: autobiographical memory, Alzheimer's, electric and magnetic stimulation

Conflicts of interests: This is my scientific review on giving brain stimulation to patients to improve memory and at the same time providing an avenue for therapy of patients with memory disorders such as Alzheimer's and dementia. This study not only helps us improvise memory but also will help us localize the brain functions and get a deeper understanding of the various parts.
FIRMST20_AB_12

Clinical Sciences - Neurology

THE POSSIBILITY OF IMPROVING MEMORY BEYOND NORMS AND PROVISION OF AN AVENUE OF THERAPY FOR MEMORY DISORDERS.

Ashwin SAIKUMAR1

1Pirogov Russian National Research Medical University, Moscow, Russian Federation

Introduction: The introduction of electrodes into various memory centres of the brain or by magnetic stimulation, it aims to improve the memory of patients with varying memory disorders and help humans achieve a heightened memory.

Materials \& Methods: Using data and experiments performed on patients while isolating those done exclusively for parts of the brain involved with episodic, semantic or autobiographical memory (AM). Also looking into patients who have a defect that gives them superior AM ( hyperthymesia) and those on the other end of the spectrum. Looking at the effects of memory caused due to stimulation both electrode and magnetic on the different parts of the brain relating to memory. Discerning ways of optimization of stimulation via electrodes.

Results: MAGNETIC: Healthy, younger and older participants on receiving a repetitive trans-cranial magnetic stimulation ( $r$-TMS), performed better on a memory task than during an $\mathrm{r}$-TMS-like placebo.

The brain stimulation reaped similar benefits for the younger and older patients. 
ELECTRIC: This kind of stimulation is done by insertion of hair-thin electrodes into the brain to deliver electricity and surge the activity of the basal nucleus of Meynert.

Using intermittent stimulation - currently not used in any application for brain in patients - the monkeys were able to remember things up to five times longer in a standard test of working memory. The optimal results were obtained at 60 pulses per second for 20 seconds followed by a $40-$ second interval without stimulation. An impairment of memory, of adult non-human primates, occurred on provision of continuous deep brain stimulation like the type used for Parkinson\&\#39;s and currently under study on humans with Alzheimer\&\#39;s.14 epilepsy patients who were scheduled to undergo surgery and required inserting intracranial depth electrodes to monitor seizure activities during the procedure, consistently remembered objects better when those objects had been paired with amygdala stimulation. There is a problem with this method as it is invasive, requiring brain surgery to implant electrodes, but it is difficult to ensure that the electrodes are placed where you need them to be, to elicit a response. Thus, optimization is key.

Conclusion: These results prove that if stimulation is optimized, there is a possibility to improve a patient's memory by localizing the area in the brain that has degraded and provision of stimulation (magnetic or electric) has the possibility to improve degenerative memory pathologies.

Keywords: electric and magnetic stimulation, Alzheimer's, autobiographical memory, memory disorders

Conflict of interests: This is my scientific review on techniques that hope to not only help patients cope with memory disorders but also help provide a deeper understanding of the brain.

\#FIRMST'20_AB_11

Clinical Sciences - Pulmonology

\section{THE UNANSWERED BIG WHY}

Ashly Roy1

1Maykop State Medical University, Maykop, Russian Federation
We sure have come a long way from trepanation and bloodletting. science have made some life changing inventions which would be mere fiction to our grandparents and great grandparents.

From measles vaccines, to restoring sight to the blindTerry Byland, we have come a long way, yet a long way to go.But, where do we stand in making life a little miserable for pulmonology patients?

Let it be asthma, emphysema, COPD, patients are leading a miserable life all across the world. its heart breaking to think that we still follow a symptomatic approach towards treating them. Doctors are trained to suppress any flare ups with corticosteroids and NSAIDS and antibiotics. Over the course of time, patients develop resistance against antibiotics, and is pushed into a complete steroid dependent phase. and are steroids any good news? By the age of fourty, patients have to live with bone weakness, sciatica, mascular dystrophy and what not. where are we making the mistake? Why does it take so long to find the underlying cause of the disease? Or why are we least bothered about finding the big WHY!

Anti-igE treatment have proved to be efficient in treating and managing allergic bronchitis, allergic bronchopulmonary aspergillosis, and asthma. I wonder how popular this option is among our doctors.

We make their life temporarily better every time they come to us asphyxiated. what we forget is, its only a matter of time until the next flare up. what we need to treat is the cause, what we need to tackle is the big WHY, and not the symptoms. its time we stop the symptomatic treatment. it s time we step up our game,find and eradicate the underlying cause and built together a better tomorrow. Thank you.

Keywords: symptomatic treatment, allergic pulmonary disorders

Conflicts of interest: even though the abstract is about the symptomatic approach towards pulmunology patients, the idea can be extended to other fields too 


\section{FÎ́RMST}

4-5 Aug 2020 | Moscow

\#FIRMST'20_AB_10

Non-Clinical Sciences - Research Methodology

\section{US DIAGNOSTICS OF CYSTIC LESIONS OF THE NECK.}

\section{Lalita YUNUSOVA1}

1Tashkent State Dental Institute, Tashkent, Uzbekistan

Neck cystic masses are fairly frequent developmental defects, and surgery is the only method of treatment, and early intervention is usually recommended to avoid complications. Materials \& Methods: 56 patients aged from 1 to 52 years (mean age $12 \pm 2$ years) with cystic neoplasms were examined. Results. Of these, on the basis of comprehensive survey, including surgical findings, 22 examined patients had thyroglossal cysts of the neck, 14 dermoid cysts of the neck, 20 had lateral cysts of the neck. thyroglossal cysts are characterized by localization in the region of the hyoid bone or near (higher or lower), while dermoid cysts are localized in the middle chordo-lingual, or chin-hyoid regions. The lateral cysts of type I were located superficially, deeper than the superficial muscle of the neck, anterior to the sternocleidomastoid (SCM) muscle. Type II was adjacent to the internal carotid artery, tightly adjacent to the internal jugular vein, and type III was located between the internal and external carotid arteries.In cystic lesions, sonography used to evaluate the localization of mass, its size, contours, internal echogenicity, the presence of septa (partitions), the presence of an artefact of distal acoustic amplification, blood flow during color Doppler mapping.Lateral cysts in US visualized in the form of cystic formations of oval or rounded shape, with smooth clear contours. In our studies, branhogenic cysts of the neck in the acute stage in 11 (19.6\%) patients on the echogram visualized as a cystic formation of an oval shape with smooth contours, clear boundaries, filled with hypoechoic containing, with a small number of isoechoic inclusions. In 5 (8.9\%) cases, the iso-echogenic area of wall thickening, edema, decrease in the echogenicity of the subcutaneous tissue and spermus muscle, which indicated the inflammatory process of the cystic neck formation, are noted at the lower pole of the formation.In $3(5.3 \%)$ patients, the festering multichamber lateral cyst of the neck was recorded on the echogram as a cystic formation, with the presence of isoechoic septa. Anechoic cyst contents acoustic artifact of amplification distal to the cyst. In 2 patients on the echogram visualized cystic formation of an oval shape with smooth contours, clear boundaries, filled with heterogeneous contents with a suspension, the effect of "pseudosolidity". In 5 (8.9\%) cases, the cyst was adjacent to the internal JV, squeezing it, as well as to the external (ECA) and (ICA). Conclusion.Radical resection of the cysts is the definite treatment to prevent recurrence.

Keywords: Cystic swelling; Neck; Ultrasonography; Dermoid cysts; Thyroglossal cysts; Lateral cysts of the neck.

Conflicts of interests: None

\#FIRMST'20_AB_9

Non-Clinical Sciences - Research Methodology

\section{SONOGRAPHY OF CYSTIC LESIONS OF THE NECK.}

\section{Lalita YUNUSOVA}

1Tashkent state dental institute, Tashkent, Uzbekistan

Neck cystic masses are fairly frequent developmental defects, and surgery is the only method of treatment, and early intervention is usually recommended to avoid complications. This study was also undertaken to assess the possibilities of ultrasound in the differentiation of cervical cysts.

Keywords: Cystic swelling; Neck; Ultrasonography; Dermoid cysts; Thyroglossal cysts; Lateral cysts of the neck.

Conflicts of interests: None 


\section{FÎ́RMST}

4-5 Aug 2020 | Moscow

\#FIRMST'20_AB_8

Non-Clinical Sciences - Molecular Biology

\section{INVESTIGATING THE ASSOCIATION BETWEEN OXYTOCIN PATHWAY POLYMORPHISMS AND THE LEVEL OF SOCIAL FUNCTIONING IN SCHIZOPHRENIA PATIENTS \\ Vera MIKHAILOVA1}

1I.M. Sechenov First Moscow State Medical University, Moscow, Russian Federation

The social adaptation for any schizophrenia patient and its family is the main factor allowing to judge whether therapy is working or not. That makes the improvement of patients' social functioning the main goal for an attending physician, the pharmaceutical industry specialists and also for biomedical researchers. To obtain a better knowledge of the pathogenesis of poor social performance in schizophrenia patients under treatment, it seems necessary to take into consideration the disturbance of the oxytocin pathway. Neurohormone oxytocin is known to play an important role in social behavior. The latest neuroimaging studies have proven OXTR (oxytocin receptor) expressing cells to be largely involved in social cognition tasks. Moreover, such polymorphisms as CD38(rs3796863), OXTR(rs53576), OXTR(rs4686302) have been observed to be associated with the pathogenesis of several diseases such as autism, OCD and bipolar disorder.

Thus, the main goal is to find the risk alleles for poor social functioning in the oxytocin pathway polymorphisms in patients with schizophrenia.

Social functioning was assessed with the Personal and the Social Performance (PSP) scale in 732 patients (women 343 ), aged $36,5 \pm 12,3$ years, with an ICD-10 diagnosis of schizophrenia or schizoaffective psychosis. Firstly, genotyping was performed with HRM (High Resolution Melt) method using DNA samples extracted from patients' venous blood samples by organic solvents and amplificated by PCR. Secondly, all patients were stratified by the level of social functioning into two groups: "moderate and good" and "marked and poor". Allele frequencies were aligned with the Hardy-Weinberg equation $(p>0,05)$. Genotypes were calculated in each group of social functioning. CC allele CD38(rs3796863) tended to be more frequent in "poor" performance group within a socially useful activity PSP scale. OXTR rs53576 GG allele was considerably more frequent in "poor" performance group within the interpersonal relations PSP scale.

It is, therefore, the first-time oxytocin pathway polymorphisms are found to be associated with social functioning in schizophrenia patients, with CD38 rs3796863 CC and OXTR rs53576 GG being risk allele.

Keywords: Oxytocin, schizophrenia, social cognition, stress factors

Conflicts of interests: no conflict of interest.

\#FIRMST'20_AB_7

Non-Clinical Sciences - Nuclear Medicine

\section{THE ROLE OF 18F-FDG PET/CT FOR THE PREDICTION OF RADIOIODINE THERAPY RESPONSE IN PATIENTS WITH METASTATIC DTC}

\section{Tamara GELIASHVILII,}

1A. Tsyb Medical Radiological Research Center Branch of the National Medical Research Radiological Center of the Ministry of Health of the Russian Federation, Obninsk, Russian Federation

The study objective was to investigate the role of $\mathrm{PET} / \mathrm{CT}$ as an indirect determination of the differentiation status of metastases and for the prediction of radioactive iodine (RAI) therapy response in patients with metastatic DTC. Materials and methods. The 40 metastatic DTC patients were enrolled in the study that underwent both posttherapeutic radioiodine scan and PET/CT at the same period. Results. 12 patients (30\%) responded to RAI therapy. The remaining 28 patients (70\%) not responded to RAl therapy showed stabilization or progression. The accumulation of radioiodine by metastases positively correlated with the total response rate (RR), while the FDG avidity is negative. Significant direct correlation with RR was observed in the group with only radioiodine uptake. However, this correlation was not observed in the patients with both tracers uptake. The patients with FDG-positive 
metastases showed poor response to RAI therapy, regardless of the degree of radioiodine uptake. Conclusion. The FDG uptake by metastases is a predictor of a poor response to RAl therapy, even in the presence of RAl uptake. The use of 18F-FDG $\mathrm{PET} / \mathrm{CT}$ in patients with metastatic DTC can be recommended at the beginning of RAl therapy to clarify the prognosis and provide a personalized approach to the treatment and observation of the most difficult category of patients.

Keywords: 18F-FDG PET/CT (PET/CT), differentiated thyroid cancer (DTC), radioiodine therapy, posttherapeutic whole-body scan (131I-WBS).

Conflicts of interests: no conflict of interest.

\#FIRMST'20_AB_6

Non-Clinical Sciences - Public Health

THE 'THOUGHT MODEL' OF SPIRITUALITY TO SOLVE COMPLEXITIES, CONFLICTS, AND AMBIGUITIES IN TRAINING AND EDUCATION OF SPIRITUAL DIMENSION OF HEALTH (SPIRITUAL HEALTH) IN MODERN PUBLIC HEALTHCARE SYSTEMS OF CULTURALLY AND RELIGIOUSLY DIVERSE SOCIETIES. Mahesh BHATT1 1MMBSHS Trust, Dehradun, India

WHO defined health as a "Complete balance of Physical, Mental, and Social health and not merely an absence of disease and infirmity?" This definition had its shortcomings, and finally, the Spiritual Dimension was added to it after the 37th World Health Assembly in 1984 after adopting a historic resolution WHA37, making the Spiritual Dimension important for the strategies for health by the WHO member states.
Multiple scientific studies have clear evidence of the importance of spiritual dimension in all domains of public health and healthcare.

In a secular democratic country like India, with its rich cultural and religious diversity, the spiritual dimension of health is often neglected because of two reasons. First, it is perceived directly in religious contexts, which make it communal, complex, ambiguous, and difficult to define, and second, its ill-defined boundaries with religions make it misfit for modern scientific healthcare systems. It resulted in neglected approach in training and application by health professionals and policymakers leading to poor research, education, and clinical use of an important dimension of health which has strong influence on the organized efforts and choices of individuals and communities in preventive, promotive, and curative health.

We proposed the 'Thought Model' of spirituality to free it from the religious bounding with a scientific approach and make it less ambiguous for public health applications based on the evolution of human thought processes. It considers the spiritual thought process as the most evolved thought of human mind in the form of humanity and human values which binds diverse religious, cultural, philosophic, and scientific thoughts across the globe at collective and individual level connecting within to its 'Self' and outwards to 'Cosmos' or 'Universe.'

In clinical settings of modern healthcare institutions, this 'thought model' of defining and understanding spirituality provides its scientific compatibility by decreasing the conflicting religious beliefs, which are common in healthcare delivery systems to almost nil by navigating the conflicting religious, cultural, and scientific thoughts. So, we can easily incorporate this model in the Curriculum of various medical and healthcare training programs.

Keywords: Spiritual health, spiritual dimension of health

Conflicts of interests: None.

\#FIRMST'20_AB_5

Clinical Sciences - Obstetrics \& Gynecology 


\section{FÎ́RMST}

4-5 Aug 2020 | Moscow

EVALUATION OF VITAMIN D STATUS IN WOMEN

DEPENDING ON THE AGE, BMI VALUES, DURATION OF DAYLIGHT HOURS AND IN POLYCYSTIC OVARIAN SYNDROME PATIENTS.

Ekaterina GAVRILOVA1

1Sechenov University, Moscow, Russian Federation

Vitamin $D$ is regulator for genes controlling the mineral homeostasis in blood and bone tissue, controlling the functions of immune system, apoptosis and other metabolic processes.

Study objective: evaluation of vitamin $D$ in a population of Russian women living in Moscow, as well as in a population of female with diagnosed polycystic ovarian syndrome PCOS along with an evaluation of the dependence of vitamin D levels in terms of age, $\mathrm{BMI}$ and duration of daylight hours.

Materials and methods. The examination performed included 753 females aged from 16 to 78 years old. Other examined population included 46 female patients 20-39 years old with diagnosed PCOS.

Results. The highest mean value of 25(OH)D was registered in a group of women aged $30-39$ years $20.1 \pm 7.4$, with the least value registered in a group aged over 70 years $-14.2 \pm 5.8$. A negative correlation was found between the vitamin $D$ levels and the age, at the same time significant decrease in the blood vitamin D levels develops in patients aged over 50 years old. Determinations of blood vitamin $D$ levels show 25(OH)D deficiency in 454 female patients (60.2\%), of which in 118 (15.6\%) the vitamin D levels were less than $10 \mathrm{ng} / \mathrm{ml}$. Insufficiency of 25(OH)D was found in 234 (31\%) females.The highest values of $25(\mathrm{OH}) \mathrm{D}$ were found in patients showing $\mathrm{BMI}$ values of $18-20.9 \mathrm{~kg} / \mathrm{m} 2$. The lowest values of vitamin $D$ were found in obese females. A positive correlation was found between the blood levels of vitamin $D$ and the duration of daylight hours. The examined population of 46 PCOS patients shows the mean vitamin D level of $17.01 \pm 6.1$. The results were compared to data from patients not diagnosed as having PCOS and aged 20-39 years old. We have discovered a decrease in the mean vitamin D levels in PCOS patients. However, significant differences between the two study groups were not found.

Conclusions: The data provided show the practicability of including high doses of vitamin D into the therapy modes for patients with PCOS and obesity, as well as during the pre- and postmenopause, regardless of the time of the year.
Keywords: Vitamin D, PCOS, obesity

Conflicts of interests: no conflict of interest.

\#FIRMST'20_AB_4

Allied Sciences - Medical Physics

NORMAL MR SPECTROSCOPIC MAP OF THE HEALTHY BRAIN

Aslanbek MIDAEV1

1iMed, Grozny, Russian Federation

1. Introduction: Magnetic resonance spectroscopy (MRS) is a method that allows to non-invasively determine the biochemical changes of certain metabolites in the brain. MR spectra characterises the metabolic processes in the specific area. The aim of this project is to create a normal MRS map of the healthy brain.

2. Methodology: For this study 15 healthy volunteers were invited. Mostly, they were of the same age and one 10 years older. That allows to compare the results of different age categories. The voxel size was taken 20 $\mathrm{mm}$, since it is the most optimal for scanning. Voxels were located on top of the corpus callosum. Data from 12 voxels was obtained and analyzed.

3. Results: The results showed that towards the center of the brain, closer to the corpus callosum, the concentration of metabolites is higher than to the cortex. Each part of the brain has individual and similar results in each patient.

4. Conclusion: This study showed the possibility of constructing MRS maps of the brain. Even the data taken from 15 patients allowed us to see the relationship between different parts of the brain. In the future, it is planned to create a more informative and complete map of patients of different age categories. This will open up new possibilities in medicine both for diagnostic and research purposes. 


\section{FÎ́RMST}

4-5 Aug 2020 | Moscow

Keywords: Metabolites, Magnetic Resonance Spectroscopy (MRS), MRS map.

Conflicts of interests: The author of this research have confirmed the lack of the conflict of interest that needs to be reported.

\#FIRMST'20_AB_3

Non-Clinical Sciences - Biotechnology

\section{DEVELOPMENT OF A NEW METHOD OF AORTIC \\ VALVE DECELLULARIZATION USING THE \\ SUPERCRITICAL EXTRACTION TECHNOLOGY. \\ Byron Simbarashe KAPOMBA1 \\ 1Sechenov University, Moscow, Russian Federation}

Valve replacement remains the last therapeutic option for patients with severe aortic valve dysfunction unsuitable for valvular reconstruction. The tissueengineered heart valve portends a new era in the field of valve replacement. One of the most promising techniques for tissue and organ regeneration is decellularization, in which extracellular matrix is isolated from its native cells and genetic material in order to produce a natural scaffold. Decellularized heart valves are of great interest as a scaffold for the tissue-engineered heart valve due to their naturally bioactive composition. One of the disadvantages of currently used decellularization protocols is long duration of the process (14 days). The object of the study was an ovine aortic root (56). The effectiveness of decellularization was evaluated by DNA quantification. Mechanical tests were carried out at EZ Test facility. Statistical processing of data was carried out by standard methods. Differences were considered significant at $p<0.05$. In contrast to the classical processing of detergents in a solution, the developed protocol made it possible to completely eliminate nuclear material. The level of residual DNA decreased from $5.14 \pm 0.01 \%$ ref. after classical treatment up to $2.70 \pm 0.22 \%$ ref. after extraction in
scCO2 medium. The hybrid treatment with detergent solutions for 24 hours and the subsequent extraction in scCO2 medium for 3 hours ( $t=37 \mathrm{C}, \mathrm{P}=15-25 \mathrm{MPa}$ ) made it possible to extract cells whilst maintaining the extracellular matrix structure. Thus, the combined treatment (detergent $+\mathrm{scCO} 2$ ) makes it possible to obtain cell-free intact matrices.

Keywords: Decellularization, aortic root, supercritical carbon dioxide,

Conflicts of interests: The need to reduce limitations of currently used aortic valve replacements.

\#FIRMST'20_AB_2

Clinical Sciences - Oncology \& Hematology

\section{SIMULTANEOUSLY DEVELOPED UTERINE CARCINOSARCOMA AND ENDOMETRIOD OVARIAN CARCINOMA IN 21-YEAR-OLD WOMAN.}

Anna, Voronova1,

1Perm State Medical University, Perm, Russian Federation

The aim: To present of a clinical case of primary multiple malignant neoplasms of the female reproductive system at a young age.

Methods: Retrospective analysis of clinical documentation, analysis of medical literature. The combination of endometrioid ovarian carcinoma and uterine carcinosarcoma in the patient is considered. 10.02.2017. The patient had complaints of cramping pains in the lower abdomen, bloody discharge from the genital tract. She was treated from 10.02.2017 to 15.02.2017 in the gynecological department, diagnosis are jected giant necrotic endometrial polyp. 10.02.2017. polypectomy. Histologically signs of malignancy were found.

From anamnesis: menstruation from 12 years, irregular, abundant. She was observed by a 


\section{FÎ́RMST}

4-5 Aug 2020 | Moscow

gynecologist with a diagnosis of endometriosis. In 2015 missed abortion. In 2015-2017 she took COC for contraceptive purposes.On 20.02.2017, she was admitted to the city hospital, hysteroscopy was performed, and the contents of the uterine cavity were aspirated. Results of histological examination: malignant tumor of the endometrium.

09.03.2017 - 27.03.2017 surgical treatment in the regional cancer center.

10.03.2017 laparotomy. Uterus and ovaries extirpation. Iliac lymph node dissection on the left. Subtotal resection of large omentum.

Histological examination of material: uterine carcinosarcoma spreading to external orifice. MTS to omentum, iliac lymph nodes on the left side. Carcinosarcoma's MTS of the right ovary. T3N1M0.

05.04.2017 - 04.09.2017 - 4 courses of chemotherapy (Paclitaxel, Carboplatin), 2 courses of radiation therapy.

14.12.2017 PET / CT: metastases of the right lung, liver, and mesenteric tissue.

Due to the lack of effect from 1st line therapy, switching to line $2 \mathrm{rd}$ line therapy.

26.12.2017 - 02.02.2018 5th,6th chemotherapy courses with second-line drugs (Docetaxel, Gemcitabine).

05.03.2018. CT: MTS to liver, peritoneum, abdominal lymph nodes, lungs, and bones (cancer progression). 13.04.2018 - 17.04.2018-chemotherapy with Pazopanib.

Immunohistochemical examination of ovaries: highlagressive endometrioid carcinoma of the right ovary.

Diagnosis: Uterus carcinosarcoma, endometrioid carcinoma of the right ovary of a high degree of malignancy. New possible therapy course should include Doxirubicin, Cisplatin (medical conclusion). The patient did not start therapy due to her severe condition after radiatio therapy and chemotherapy. The patient has progressive ascites, laparocentesis was performed on 01.08.2018. 06.08.2018 a patients has died without regaining consciousness.The relatives refused the pathological examination.

Summary: Diagnosis and treatment of reproductive system primary multiple malignant neoplasms in young patients with complicated gynecological anamnesis is an extremely difficult task. The combination of T3N1M0 uterine carcinosarcoma with highly-agressive endometrioid ovarian carcinoma resulted in a fatal outcome despite the combined treatment.

Keywords: Carcinosarcoma of uterus, endometrial ovarian carcinoma.

No conflict of interest: Conflicts of interests: 


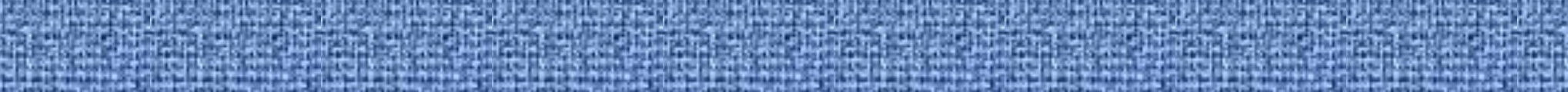

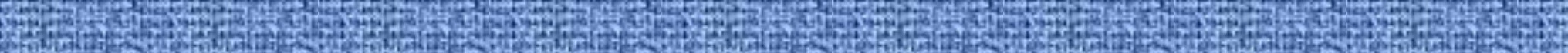

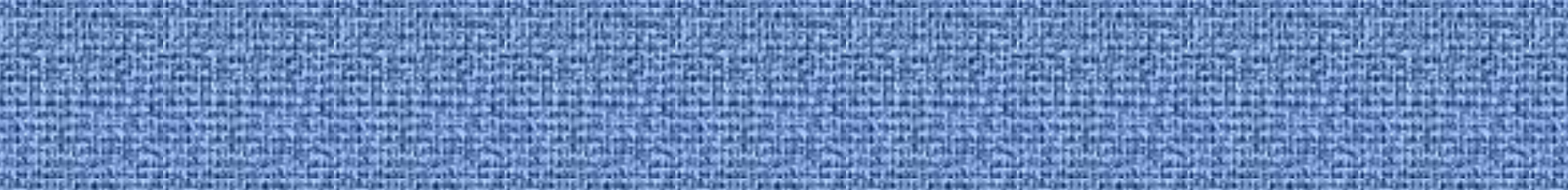

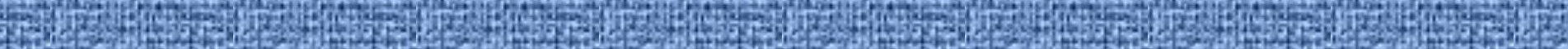
4.5.

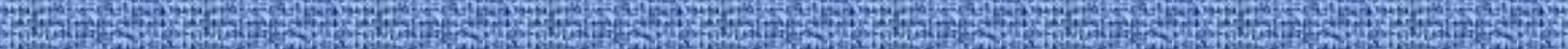

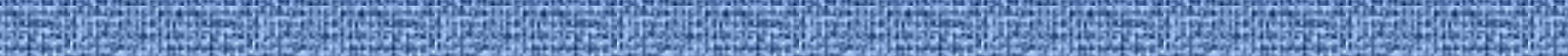
1.

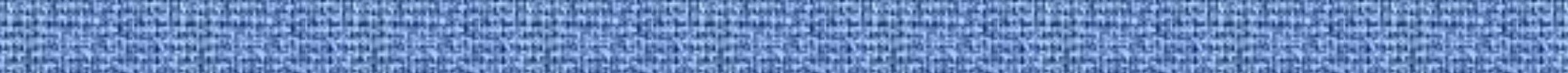

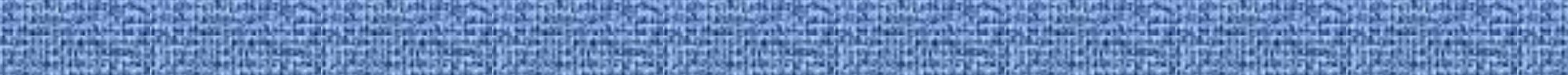
1.120.

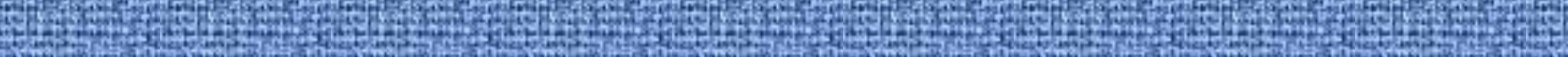
TH

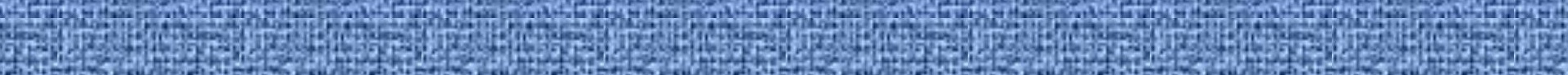

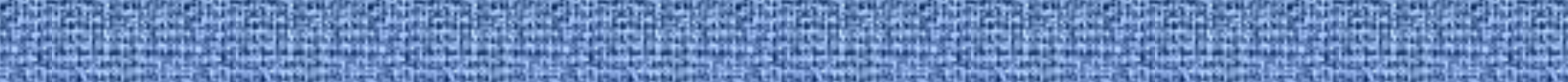
STH

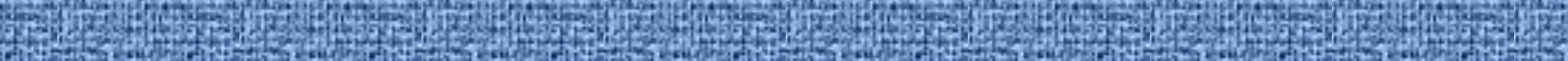

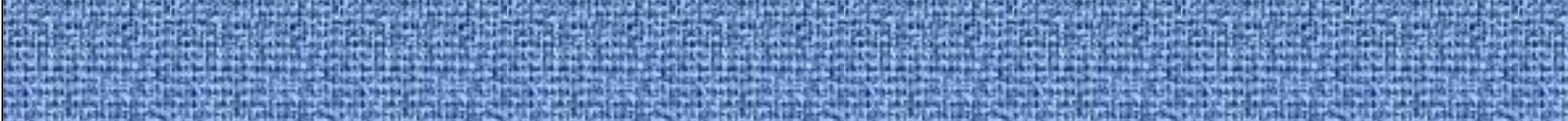

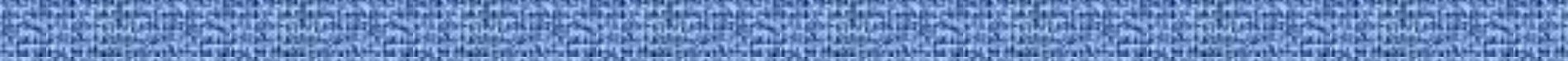

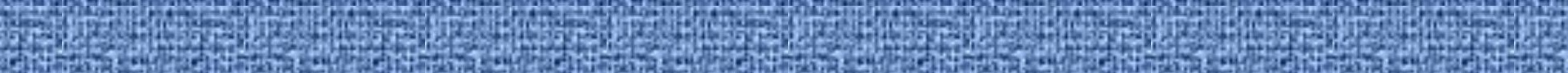
47. A Th 1.7. 32tor

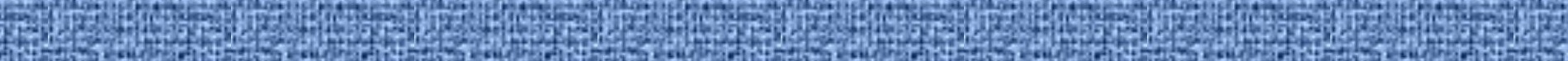

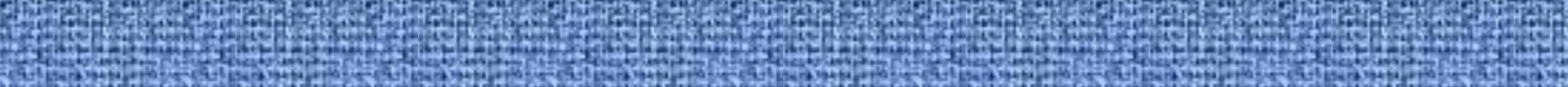

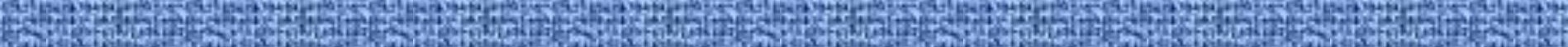

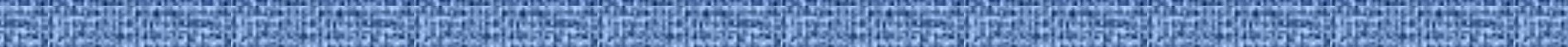
4 4. 20.7.

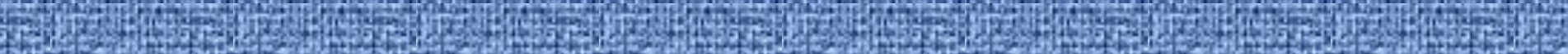
19. 